\title{
De la modélisation à la commande non linéaire des convertisseurs multicellulaires série. Application à la fonction hacheur
}

\author{
G. Gateau (*), P. Maussion et T. Meynard \\ Laboratoıre d'Électrotechnıque et d'Électronıque Industrielle $\left(^{* *}\right)$, BP 7122 , \\ 2 rue Charles Camichel, 31071 Toulouse Cedex 7, France
}

(Reçu le 24 octobre 1996, révisé le 20 février 1997, accepté le 10 mars 1997)

\begin{abstract}
PACS 07.05 -t - Computers in experimental physics
PACS 07.50.-e - Electrical and electronic components, instruments and techniques

PACS 02.90.+p - Other topics in mathematical methods in physics
\end{abstract}

\begin{abstract}
Résumé. - La commande des convertısseurs statiques à structure classique est un domaine qui est à l'heure actuelle bien maîtrisé et a été largement exploré par de nombreux chercheurs au cours de ces dernıères années. Avec l'arrıvée de nouvelles structures de convertisseurs (multicellulaures), de nouveaux problèmes en termes de commande sont apparus. Nous présenterons dans cet article une nouvelle approche développée pour la commande d'un convertisseur multıcellularre en association sérıe Après quelques rappels sur la structure et l'aspect modélisation de ce type de convertisseur, nous présenterons une procédure de régulation utılisant le principe de lınéarisation exacte entrées/sorties. Nous termınerons cet article par la validation de ce type de commande à l'aide de sımulations prenant en compte les problèmes d'échantillonnage et d'implantation numérıque
\end{abstract}

\begin{abstract}
Nowadays, the linear control of classical static power converters is a well known ground, almost fully investigated by numerous researchers all around the world. Besides, some new control problems appeared with new power converter architectures, such as multi-cell converters. In this paper a new method is presented for the control of a series multi-level converter, by the mean of input-output linearization. First, the multi-level structure and modelling are recalled, then the principle of the voltage and current control using exact input-output linearization, is presented. The sample period and the digital implementation problems will be taken into account for complete validation of this type of new control.
\end{abstract}

\section{Introduction}

\subsection{La MOdÉLISATION DES CONVERTISSEURS MULTICELlulaires EN ASSOCiation SÉRIE}

L'aspect modélisation dans l'étude d'une commande de convertisseur statique revêt un intérêt tout particulier. En effet, un convertisseur statique (multi-niveaux ou non) possède des variables continues (généralement courants et tensions) mais aussi des variables discontinues (états des interrupteurs).

$\left({ }^{*}\right)$ Auteur auquel doit être adressée la correspondance (e-mall · (gateau, maussion)@leei enseeiht fr) $\left({ }^{* *}\right)$ UPRESA C N.R.S. 5004

(C) Les Éditions de Physique 1997 
En électrotechnique, on utilise souvent un modèle aux valeurs moyennes instantanées. Pour ces modèles, on remplace les grandeurs discontinues par leurs valeurs moyennes glissantes sur une période de découpage. Cette modélisation est tout à fait justifiée et justifiable tant que la période de découpage est inférieure aux constantes de temps mises en jeu par le système. Les discontinuités (changements d'état des interrupteurs) conduisent à la génération d'harmoniques haute fréquence qui, en général, ne modifient pas qualitativement le fonctionnement propre du système. Le cas des convertisseurs multicellulaires est un peu particulier car la génération de ces harmoniques à la fréquence de découpage et au-delà, entraîne un équilibrage naturel des tensions appliquées aux bornes des interrupteurs bloqués $[1,4]$. Cependant, ce phénomène reste généralement assez lent et peut être négligé lorsqu'on envisage une régulation active de ces tensions, ce qui est le cas dans cet article.

Nous allons décrire rapidement les différents modèles de représentation possibles pour les convertisseurs statiques multicellulaires :

\section{- Modèle direct :}

Ce modèle représentera fidèlement l'état de chacune des cellules du convertisseur à l'échelle de la période de découpage. Tous les phénomènes harmoniques ou haute fréquence seront donc présents dans ce modèle. Il sera nommé modèle Direct ou Réel et nous servira uniquement pour la validation des lois de commande synthétisées à partir d'un modèle aux valeurs moyennes.

- Modèle aux valeurs moyennes :

Ce modèle continu dans lequel toutes les grandeurs sont constantes durant une période de découpage nous servira à faire la synthèse de la commande des systèmes multicellulaires.

\section{- Modèle harmonıque :}

Ce modèle ne sera pas étudié dans cet article. Il permet néanmoins d'obtenir une représentation dynamique d'un convertisseur multicellulaires en prenant en compte tous les phénomènes harmoniques [9].

1.2. But DE L'ÉTUdE. - Le but de l'étude sera d'abord de présenter le principe de modélisation pour les convertisseurs multicellulaires en association série. À partir d'un modèle non linéaire, nous effectuerons la commande des variables d'état du système en utilisant la méthode de linéarisation exacte entrées/sorties. Nous étudierons ensuite l'effet de la période d'échantillonnage et du temps de calcul sur l'algorithme présenté en vue d'une implantation de ce type de commande sur une maquette expérimentale.

\section{Modélisation}

2.1. SChÉma D'ÉTUde, MISE EN SÉRIE. - Nous allons au cours de cette section, présenter les modèles actuellement disponibles pour représenter l'évolution dynamique des systèmes multicellulaires $[1,3]$. Ces modèles seront tous basés sur une représentation aux valeurs moyennes des dispositifs. Cette représentation n'est pas la seule ni la meilleure, néanmoins elle nous permet le développement de lois de commande tout en représentant assez fidèlement le comportement dynamique du système.

Après le principe de la mise en série, nous présenterons la structure interne d'une cellule puis les différents modèles utilisés. 


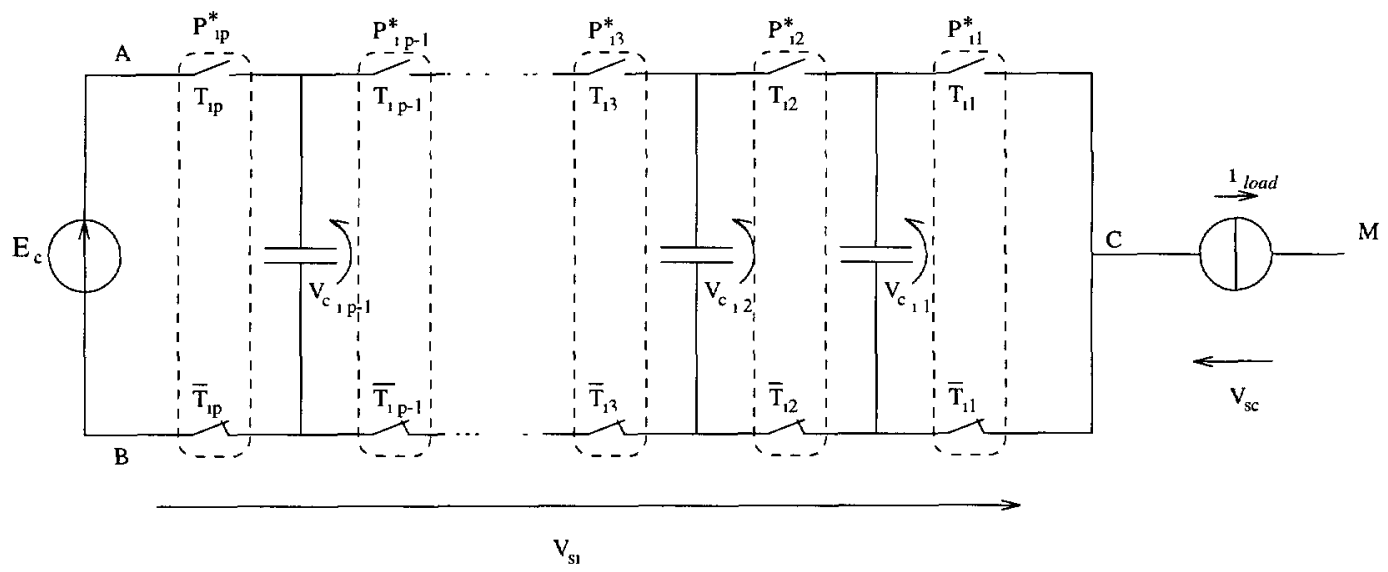

Fig 1. - Schéma général : association série.

[General scheme with serial association]

2.1.1. Système multicellulaire série. - La figure 1 représente une association série de $p$ cellules de commutation Chaque cellule est formée d'une paire d'interrupteurs $\left(T_{\imath k}, \bar{T}_{\imath k}\right)$ dont l'état est complémentaire. L'ensemble des cellules constitue un "bras". On pourra associer plusieurs bras en parallèle pour obtenir un fonctionnement en onduleur monophasé ( 2 bras) ou triphasé (3 bras).

On remarque qu'entre chaque cellule est inséré un condensateur flottant. En régime permanent, chaque condensateur voit une tension égale à $k e_{\mathrm{c}} / p$, où $k$ représente la position du condensateur dans l'association et $p$ le nombre de cellules.

Les différentes notations utilisées dans la figure 1 sont définies en annexe.

La tension d'alimentation $E_{\mathrm{c}}$ sera fournie au travers d'un filtre d'entrée de type $L C$. La charge sera connectée entre les points $\mathrm{C}$ et $\mathrm{M}$ et sera pour cette étude de type $R L$.

Pour un fonctionnement de type hacheur, le point $M$ sera connecté à $B$ et à la masse de l'alimentation de puissance. Pour un fonctionnement en onduleur avec point milieu capacitif, le point $M$ sera relié au point milieu de l'alimentation. Enfin, pour un fonctionnement en onduleur en pont complet, ce point sera connecté à un deuxième bras.

2.1.2. Étude de l'association de deux cellules. - Afin de définir les notations utilisées, nous avons représenté sur la figure 2 , deux cellules avec leur condensateur flottant.

On pose l'indice $\imath$, le numéro du bras étudié et l'indice $k$, le numéro de la cellule étudiée.

L'état de la cellule $k$ du bras $i$ sera représenté par $P_{\imath k}^{\star}(t)$. On note $T_{\imath k}$ et $\bar{T}_{\imath k}$ l'état des deux interrupteurs constituant la cellule $k\left(T_{2 k}=0 \rightarrow\right.$ interrupteur OFF et $T_{2 k}=1 \rightarrow$ interrupteur $\mathrm{ON})$. L'état interne de ces deux interrupteurs étant complémentaire, on aura donc :

$$
\left\{\begin{array}{llll}
P_{2 k}^{\star}(t)=0 & \rightarrow T_{2 k}(t)=0 & \text { et } & \bar{T}_{2 k}(t)=1 \\
P_{2 k}^{\star}(t)=1 & \rightarrow T_{\imath k}(t)=1 & \text { et } & \bar{T}_{2 k}(t)=0
\end{array} \quad \text { avec } \quad \begin{array}{l}
k \in[1 \ldots p] \\
i \in\left[1 \ldots n_{\mathrm{b}}\right]
\end{array}\right.
$$

2.1.3. Mise en équations du système. - D'après la figure 1 , la tension $V_{s_{2}}(t)$ est la somme des tensions aux bornes des cellules $P_{\imath k}^{\star}$ et peut être exprimée par l'équation (2). On remarque donc que cette tension dépend des différences de potentiel entre les différentes cellules, c'est-à-dire 


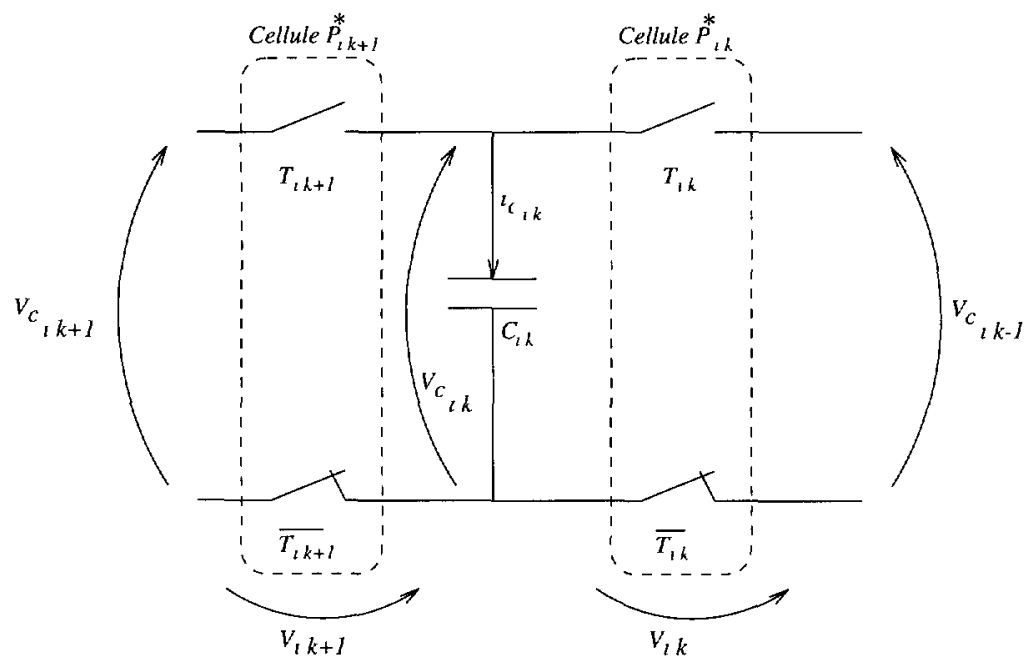

Fig. 2 - Association de 2 cellules.

[Association of two cells.]

aux bornes des condensateurs flottants .

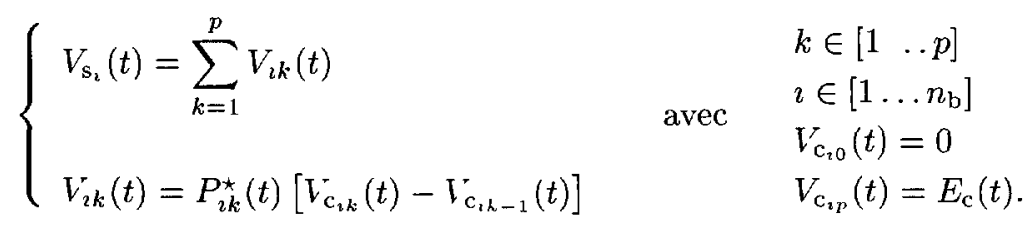

La tension $V_{\mathrm{s}_{2}}(t)$ peut également s'exprimer sous la forme de l'équation (3) où l'on peut remarquer cette fois qu'elle dépend des différences de commande appliquées sur chaque bras :

$$
V_{\mathrm{s}_{2}}(t)=\sum_{k=1}^{p} V_{\mathrm{c}_{, k}}(t)\left[P_{\imath k}^{\star}(t)-P_{\imath k+1}^{\star}(t)\right] \quad \text { avec } \quad \begin{aligned}
& k \in[1 \ldots p] \\
& \\
& \quad P_{\imath p+1}^{\star}(t)=0 \\
& V_{\mathrm{c}_{\imath p}}(t)=E_{\mathrm{c}}(t)
\end{aligned}
$$

Suivant la structure du circuit (point milieu, pont complet, ...), on calculera la tension $V_{\mathrm{sc}}(t)$ aux bornes de la charge à partır de son expression en fonction des tensions $V_{\mathrm{s}_{\mathbf{z}}}(t)$.

On étudie l'expression du courant dans le condensateur $k$ du bras $i$, c'est-à-dire $i_{\mathrm{C}_{1 k}}(t)$. On supposera que les interrupteurs sont parfaits, donc que l'équation (1) est vérifiée. On montre facilement que pour obtenir un courant non nul dans une cellule, il suffit que deux cellules successives aient un état différent sinon le courant est nul :

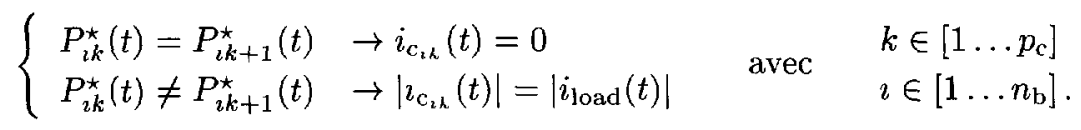

Pour obtenir le signe de $i_{c_{i k}}(t)$, on doit considérer l'état des interrupteurs $k$ et $k+1$ comme le montre l'équation (5). 
On peut donc écrire :

$$
\forall t \geq 0, \quad i_{\mathrm{c}_{\imath k}}(t)=\left[P_{\imath k+1}^{\star}(t)-P_{\imath k}^{\star}(t)\right] i_{\mathrm{load}}(t) \quad \text { avec } \quad \begin{aligned}
& k \in\left[1 \ldots p_{\mathrm{c}}\right] \\
& i \in\left[1 \ldots n_{\mathrm{b}}\right] .
\end{aligned}
$$

L'écriture du courant condensateur (5) nous permet maintenant d'exprimer l'évolution de la tension aux bornes du condensateur de la cellule $k$ du bras $i$.

$$
\begin{array}{rlrl}
\imath_{\mathrm{c}_{\imath k}}(t) & =C_{\imath k} \frac{\mathrm{d} V_{\mathrm{c}_{\imath k}}(t)}{\mathrm{d} t} \\
\text { d'où } \quad \dot{V}_{\mathrm{c}_{2 k}}(t) & =a_{\imath k} i_{\text {load }}(t)\left[P_{\imath k+1}^{\star}(t)-P_{\imath k}^{\star}(t)\right] . & & \text { avec } \quad a_{\imath k}=\frac{1}{C_{\imath k}}
\end{array}
$$

La première remarque intéressante est que chaque tension condensateur sera commandée par la différence des états de chaque cellule encadrant ce condensateur. Il est à noter que cette commande dépend fortement du courant circulant dans la charge.

Toutes les équations précédentes sont valables à tout instant, elles représentent l'évolution de l'état du système. Le désavantàge évident de ce type de représentation est qu'elle est discontinue puisqu'avec les hypothèses posées (interrupteur parfait), l'état des cellules passe de 0 à 1 en un temps nul.

Pour contourner ce problème, nous effectuerons une modélisation aux valeurs moyennes du système, en supposant faible la période de découpage par rapport aux constantes de temps mises en jeu dans le système.

On utilisera comme grandeurs représentatives des courants, des tensions et des états des cellules, leurs valeurs moyennes sur une période de découpage.

Ainsi on posera :

$$
\begin{aligned}
& u_{\imath k}(t)=\left\langle P_{\imath k}^{\star}(t)\right\rangle \\
& v_{\mathrm{c}_{2 k}}(t)=\left\langle V_{\mathrm{c}_{2 k}}(t)\right\rangle \\
& e_{\mathrm{c}}(t)=\left\langle E_{\mathrm{c}}(t)\right\rangle \\
& \imath_{\ell}(t)=\left\langle i_{\text {load }}(t)\right\rangle
\end{aligned}
$$

où $e_{\mathrm{c}}(t)$ représente la valeur moyenne de $E_{\mathrm{c}}(t)$ sur une période de découpage.

Nous utiliserons toujours la même représentation pour le vecteur d'état modélisant un système multi-niveaux. Pour simplifier les notations, le vecteur d'état intégrant toutes les grandeurs dépendant du temps sera noté :

$$
X(t)=X=\left[x_{1}, x_{2}, \ldots, x_{\imath}, \ldots, x_{n}\right]^{t} .
$$

Il sera donc composé des tensions condensateurs pour chaque bras puis des courants pour chaque bras.

On obtient donc pour un hacheur 3 cellules le vecteur d'état :

$$
X=\left[x_{1}, x_{2}, x_{3}\right]^{t}=\left[v_{\mathrm{c}_{1}}, v_{\mathrm{c}_{2}}, i_{\ell}\right]^{t}
$$

et comme vecteur de commande $u=\left(u_{11}, u_{12}, u_{13}\right)^{t}$ que l'on notera donc $u=\left(u_{1}, u_{2}, u_{3}\right)^{t}$ puisqu'un seul bras sera utilisé. Pour mettre en place ces notations, nous allons rechercher le modèle complet pour un hacheur 3 cellules. 


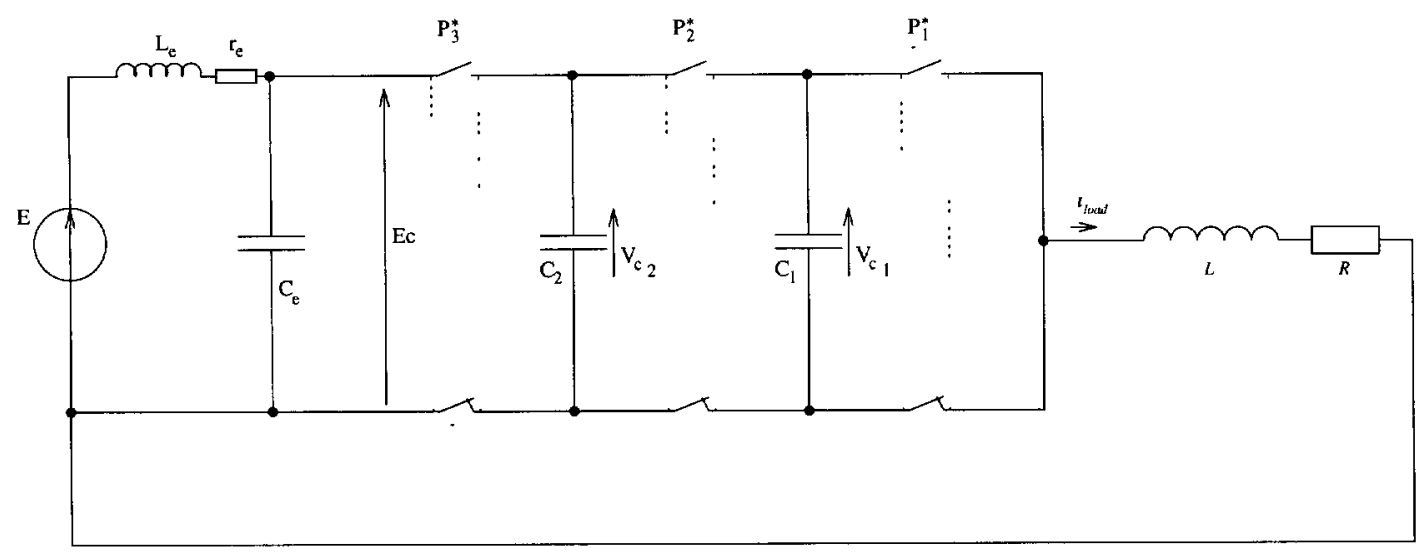

Fig. 3. - Hacheur 3 cellules

[3 cells DC to DC converter ]

\subsection{Association des Cellules en SÉrie, CAS D'un hacheur 3 Cellules}

2.2.1. Équations d'état du système. Représentation non linéarre affine. - La figure 3 représente un hacheur 3 cellules avec un filtre d'entrée.

Le comportement d'un hacheur 3 cellules est décrit par le système d'équations (8).

$$
\begin{aligned}
& u_{1}, u_{2}, u_{3} \in[0 \ldots 1] \\
& \dot{v}_{\mathrm{c}_{2}}=\dot{x}_{2}=-a_{2} x_{3}\left(u_{2}-u_{3}\right) \\
& \dot{\mathrm{i}}_{\ell}=\dot{x}_{3}=-b_{0} x_{3}+b_{1} v_{\mathrm{s}_{1}}
\end{aligned}
$$

avec

$$
\begin{array}{ll}
a_{1}=\frac{1}{C_{1}} & a_{2}=\frac{1}{C_{2}} \\
b_{0}=\frac{R}{L} & b_{1}=\frac{1}{L}
\end{array}
$$

On va maintenant mettre le système sous une forme de représentation non linéaire affine, ce qui va nous permettre pour la suite d'envisager des méthodes de découplage non linéaires. Cette écriture nous conduit à mettre le système sous la forme .

$$
\dot{X}=f_{3}(X)+\sum_{k=1}^{3} g_{3_{h}}(X) u_{k} .
$$

On pose donc :

$$
f_{3}(X)=\left[\begin{array}{lll}
0, & 0, & -b_{0} x_{3}
\end{array}\right]^{t}
$$


et

$$
g_{3}(X)=\left[\begin{array}{ccc}
-x_{3} a_{1} & x_{3} a_{1} & 0 \\
0 & -x_{3} a_{2} & x_{3} a_{2} \\
b_{1} x_{1} & b_{1}\left(x_{2}-x_{1}\right) & b_{1}\left(e_{c}-x_{2}\right)
\end{array}\right]
$$

La matrice $g_{3}(X)$ se décompose en 3 colonnes, chacune de ces colonnes représentera une fonction $g_{3_{\curlywedge}}(X)$ pour $k \in[1 \ldots 3]$. Ainsi la fonction $g_{3}(X)$ s'écrit :

$$
g_{3}(X)=\left[g_{3_{1}}(X), g_{3_{2}}(X), g_{3_{3}}(X)\right]
$$

Cette représentation nous montre bien que le système est non linéaire mais aussi que les nonlinéarités qui interviennent sont simplement dues au couplage des commandes. On pourra donc applıquer sur ce modèle une méthode de linéarisation entrées/sorties.

2.3. Utilisation DU MODÈle AUX VAleurs MOYEnNes pour la Simulation. - Tous les modèles développés au cours de cette première partie sont des modèles de représentation volontairement simplifiés des convertisseurs multicellulaires en associations série. En effet, seule la valeur moyenne des signaux est prise en compte pour la synthèse de la loi de commande.

Il est alors légitime de se poser la question de savoir ce que devient le système réel avec sa boucle de régulation lorsque que l'on prend en compte dans le modèle direct toutes les commutations. Pour cela plusieurs méthodes sont à notre disposition.

La première, de loin la plus simple mais également la plus coûteuse en temps de calcul, consiste à générer les ordres de commande pour chaque cellule de façon à obtenir à l'échelle de la période de découpage, l'état de chaque cellule que l'on a déjà appelé $P_{k}^{\star}(t)$. Le problème est que l'on fait intervenir des phénomènes ayant des constantes de temps très différentes. D'une part les commutations des cellules imposent des pas de simulation extrêmement petits (inférieur à la micro-seconde) pour obtenir une bonne précision alors que d'autre part la boucle de commande se contente de pas de simulation de l'ordre de la centaine de micro-secondes seulement.

Ce que nous allons maintenant présenter est la méthode utilisée pour générer les ordres de commande. On obtiendra donc une simulation du modèle direct du dispositif. Les seules hypothèses qui subsistent dans ce cas sont que les interrupteurs et tous les composants sont parfaits.

Nous utiliserons donc ce moyen de simulation appelé simulation directe pour valider les lois de commande synthétisées à partir du modèle aux valeurs moyennes.

La figure 4 précise le mode de génération des ordres de commande. On génère d'abord $p$ porteuses triangulaires décalées de $2 \pi / p$. Les porteuses ont des valeurs comprises entre 0 et 1. La commande génère $p$ modulantes avec une profondeur de modulation donnée. Après comparaison entre la porteuse et la modulante, on en déduit l'état respectif de chacune des cellules de commutation.

\section{Régulation non linéaire}

3.1. LiNÉARISATION ENTRÉES/SORTIEs. - Après avoir formalisé les modèles de représentation des systèmes multi-niveaux, nous allons maintenant présenter une méthodologie afin 

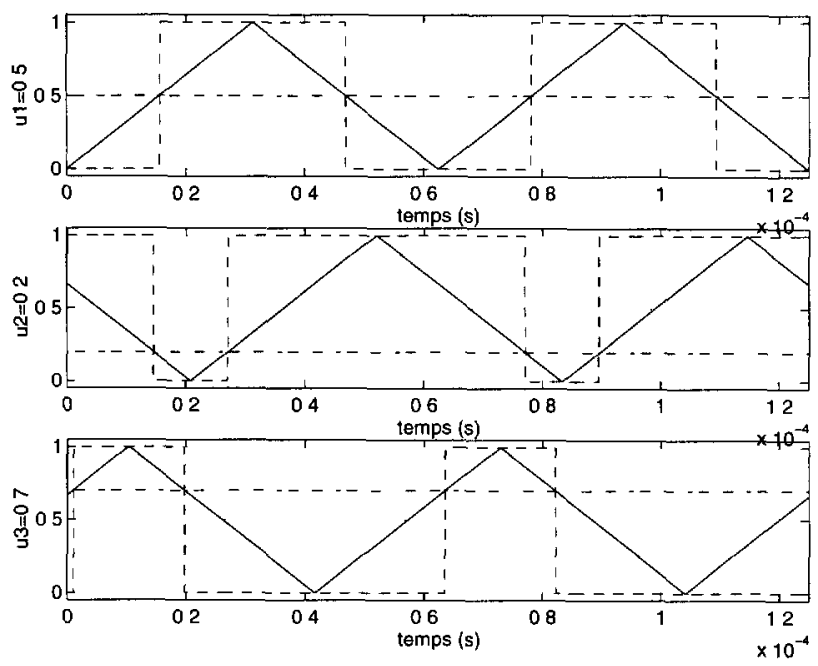

Fig. 4. - Générations des ordres de commande pour 3 cellules.

[Control signal generation for 3 cells.]

d'étudier une commande pour ce type de système. L'étude des différents modèles nous a montré qu'un système multicellulaire possède des non-linéarités principalement dues au fait que les commandes pour chaque cellule ne sont pas indépendantes. Ceci ne représente pas une forte non-linéarité mais nous oblige à envisager une commande découplante pour ce système.

Pour étudier une commande classique de type linéarisation entrées/sorties, plusieurs solutions s'offrent à nous. La première est de faire une linéarisation approchée autour d'un point d'équilibre du système, c'est-à-dire un découplage linéaire d'un modèle linéarisé du système. Cette approche est intéressante et se prête bien aux problèmes de régulation. Elle possède néanmoins l'inconvénient de limiter les excursions du point d'équilibre sous peine de perdre la validité du modèle linéarisé.

La deuxième, qui est nommée linéarisation exacte entrées/sorties permet elle de grandes transitions du point d'équilibre, mais en revanche cette méthode peut faire apparaître des problèmes de singularités. Il est important de noter que quelle que soit la méthode choisie, l'état du système devra être mesuré.

Nous avons choisi d'appliquer dans cette première étude la méthode exacte ce qui nous permettra d'avoir une commande indépendante du point de vue fonctionnement, même si certains problèmes de singularité seront à résoudre. L'intérêt est également la portée de la méthode suivant la structure (onduleur, hacheur, ...).

De plus, l'application d'une méthode classique nous permettra de bien mettre en évidence tous les problèmes relatifs à la commande des systèmes multicellulaires.

3.1.1. Généralités. - La linéarisation exacte [7,8] est une approche pour la construction de correcteurs non linéaires. L'idée principale de cette méthode est d'effectuer une transformation algébrique d'un système dynamique non linéaire, en un système totalement ou partiellement linéaire. Ainsi après transformation, toutes les technıques de synthèse de correcteurs linéaires peuvent être appliquées. L'intérêt de cette méthode est qu'elle effectue une transformation exacte du système d'état non linéaire, sans passer par une approximation linéaire comme les techniques de linéarisation classiques (jacobien) [7]. 
La linéarisation exacte d'un système peut donc être vue comme une méthode pour transformer un modèle de système vers un modèle ayant une forme plus simple. Cette méthode a été utilisée avec succès dans de nombreuses applications $[5,6,11-13]$. Néanmoins, un nombre important d'inconvénients et de limitations est lié à cette méthode comme nous le verrons au cours de cette étude.

Après quelques rappels nécessaires, nous appliquerons cette méthode au découplage.d'un hacheur 3 cellules.

\subsubsection{Linéarisation exacte entrées/sorties}

Définition du système.- Soit $(\Sigma)$, un système non linéaire affine multi-entrées, multi-sorties (MIMO), quı peut être représenté par les équations d'état (14) :

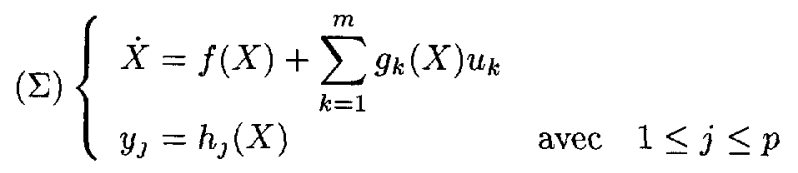

où $X \in \Omega$ est le vecteur d'état avec $\Omega \subset \mathbb{R}^{n}$ et $m$ le nombre d'entrées. Les fonctions $f$, $g_{1}, \ldots, g_{k}$ sont des applications vectorielles de $\Omega$ dans $\mathbb{R}^{n}$ et les fonctions $h_{\jmath}$ sont des fonctions de valeurs réelles définies sur $\Omega$. Nous nous intéresserons plus particulièrement aux systèmes carrés donc on posera $p=m$.

Une représentation vectorielle peut être donnée par :

$$
f(X)=\left(\begin{array}{c}
f_{1}(X) \\
\vdots \\
f_{n}(X)
\end{array}\right), g_{k}(X)=\left(\begin{array}{c}
g_{1 k}(X) \\
\vdots \\
g_{n k}(X)
\end{array}\right), \text { et } h(X)=\left(\begin{array}{c}
h_{1}(X) \\
\vdots \\
h_{m}(X)
\end{array}\right)
$$

Les éléments de $f, g_{k}, h$ sont des fonctions lisses, c'est-à-dire que ces fonctions sont indéfiniment dérivables par rapport à chacun de leurs arguments.

Dérivée de Lie.- Étant donnée une fonction lisse $h_{\jmath}(X)$, le gradient de $h_{\jmath}$ est noté $\nabla h_{\jmath}$ et s'exprime par :

$$
\nabla h_{\jmath}=\frac{\partial h_{\jmath}}{\partial X}
$$

Ce gradient est représenté par un vecteur colonne d'éléments $\left(\nabla h_{\jmath}\right)_{k}=\frac{\partial h_{\jmath}}{\partial x_{k}}$.

De la même façon, étant donné un champ de vecteurs $f(X)$, le jacobien de $f$ est noté $\nabla f$ et s'exprime par

$$
\nabla f=\frac{\partial f}{\partial X}
$$

Le jacobien est une matrice $n \times n$ qui a pour éléments $(\nabla f)_{k \jmath}=\frac{\partial f_{k}}{\partial x_{j}}$.

On pose maintenant une fonction scalaire $h_{J}(X)$ lisse et un champ de vecteurs lisse $f(X)$. On définit une nouvelle fonction scalaire $L_{f} h_{\jmath}$ appelée dérivée de Lie de $h_{\jmath}$ par rapport à $f$ comme suit :

$$
L_{f} h_{\jmath}(X)=\sum_{k=1}^{m} \frac{\partial h_{\jmath}(X)}{\partial x_{k}} f_{k}(X)=\frac{\partial h_{\jmath}(X)}{\partial X_{\imath}} f(X)
$$


Résultat général. - En reprenant le modèle du système donné par (14), on peut écrire la première dérivée de $y_{3}$ à l'aide des dérivées de Lie :

$$
\dot{y}_{j}=\frac{\partial h_{\jmath}(X)}{\partial X} \dot{X}=\frac{\partial h_{\jmath}(X)}{\partial X}\left(f(X)+\sum_{k=1}^{m} g_{k}(X) u_{k}\right) .
$$

On obtient alors

$$
\dot{y}_{\jmath}=L_{f} h_{\jmath}(X)+\sum_{k=1}^{m}\left(L_{g_{k}} h_{\jmath}(X)\right) u_{k}
$$

Il est intéressant de noter que si $L_{g_{k}} h_{\jmath}(X)=0$, les entrées $u_{k}$ n'ont pas d'influence sur les sorties.

On note ainsi $r_{J}$ le plus petit nombre entier pour qu'une des entrées $u_{k}$ apparaisse dans la $r_{J}$--ième dérivée de la sortie $y_{j}$. On a alors :

$$
y_{\jmath}^{\left(r_{\jmath}\right)}=L_{f}^{\left(r_{3}\right)} h_{\jmath}(X)+\sum_{k=1}^{m} L_{g_{\curlywedge}}\left(L_{f}^{\left(r_{3}-1\right)} h_{\jmath}(X)\right) u_{k} .
$$

Les nombres obtenus $r_{3}$ sont appelés les degrés relatifs.

On définit la matrice de découplage $m \times m, \Delta(X)$ comme suit :

$$
\Delta(X)=\left(\begin{array}{lll}
L_{g_{1}} L_{f}^{\left(r_{1}-1\right)} h_{1}(X) & . & L_{g_{m}} L_{f}^{\left(r_{1}-1\right)} h_{1}(X) \\
\vdots & \ddots & \vdots \\
L_{g_{1}}^{c} L_{f}^{\left(r_{m}-1\right)} h_{m}(X) & \ldots & L_{g_{m}} L_{f}^{\left(r_{m}-1\right)} h_{m}(X)
\end{array}\right)
$$

On définit également le vecteur $\Delta_{0}(X)$ par :

$$
\Delta_{0}(X)=\left(\begin{array}{c}
L_{f}^{\left(r_{1}\right)} h_{1}(X) \\
\vdots \\
L_{f}^{\left(r_{m}\right)} h_{m}(X)
\end{array}\right)
$$

Il est alors possible de réécrire l'équatıon (21) en utilisant (22) et (23):

$$
Y=\left(\begin{array}{c}
y_{1}^{\left(r_{1}\right)} \\
\vdots \\
y_{m}^{\left(r_{n_{1}}\right)}
\end{array}\right)=\Delta_{0}(X)+\Delta(X)\left(\begin{array}{c}
u_{1} \\
\vdots \\
u_{m}
\end{array}\right)
$$

- Théorème :

a) Le système $(\Sigma)$ est découplé sur $\Omega \subset \mathbb{R}^{n}$ si et seulement si :

$$
\operatorname{rang} \Delta(X)=m \quad \forall X \in \Omega
$$

b) Si cette condition est satisfaite alors le retour d'état non-linéaire

$$
u(X)=\alpha(X)+\beta(X) v
$$




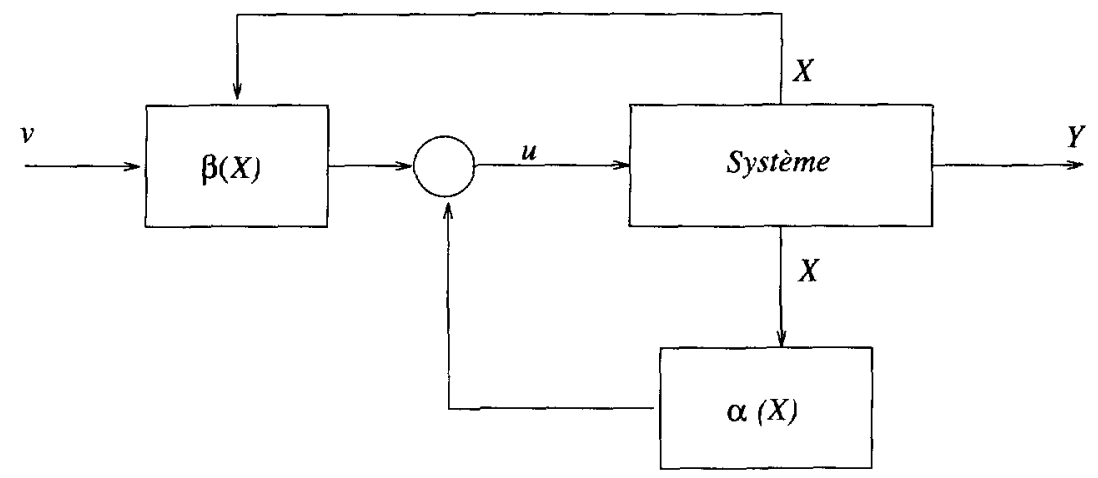

Fig. 5. - Représentatıon du découplage par linéarisatıon exacte.

[System with input/output linearization.]

avec

$$
\left\{\begin{array}{l}
\alpha(X)=-\Delta^{-1}(X) \Delta_{0}(X) \\
\beta(X)=\Delta^{-1}(X) \\
\text { et } v \text { le nouveau vecteur d'entrée }
\end{array}\right.
$$

découple le système $(\Sigma)$ sur $\Omega$.

c) Le système bouclé $(h, f+g \alpha, g \beta)$ possède un comportement entrées/sorties linéaire décrit par :

$$
y_{\jmath}^{\left(\boldsymbol{r}_{\jmath}\right)}=v_{\jmath} \quad \forall j \in[1, \ldots, m] .
$$

On effectue sur le système ainsi linéarisé (Fig. 5) par ce retour d'état non linéaire un second bouclage linéaire afin d'imposer au système la dynamique désirée. Toutes les méthodes de synthèse de correcteurs linéaires pourront ainsi être utilisées.

Il est important de noter que cette méthode aboutit à un découplage total si on a $\sum r_{\jmath}=n$, c'est-à-dire que le système découplé est linéaire pour toutes les variables d'état.

Si on observe $\sum r_{J}<n$, on obtient alors $n-\sum r_{\jmath}$ dynamiques de zéros qui conduisent à une étude plus complexe (étude des dynamiques de zéros).

On obtient après découplage le schéma de la figure 6 .

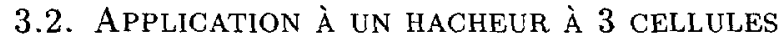

3.2.1. Application du calcul du découplage. Traitements des singularités

Modèle et paramètres du système. - Nous allons maintenant appliquer cette méthode à un hacheur 3 cellules. Le vecteur d'état du système est donc d'ordre 3 et se trouve composé des deux tensions $v_{\mathrm{c}_{1}}$ et $v_{\mathrm{c}_{2}}$ et du courant dans la charge $\imath_{\ell}$.

Le modèle utilisé pour le hacheur sera donc un modèle non linéaire affine donné par l'équation (10).

Les entrées $u_{k}$ symbolisent les rapports cycliques moyens pour chaque cellule et les fonctions $f_{3}(X)$ et $g_{3}(X)$ sont données par les équations (11) et (12).

Les fonctions $g_{3_{k}}(X)$ représenteront alors les colonnes de la matrice $g_{3}(X)$. De plus nous avons supposé que toutes les tensions condensateurs sont mesurées, donc que la fonction $h_{3}(X)$ 

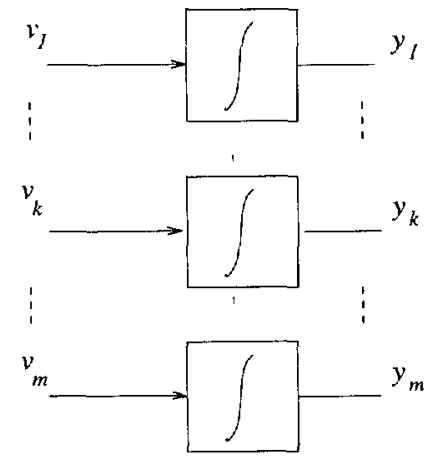

Fig. 6 - Système découplé.

[Decoupled system ]

peut être exprimée par :

$$
h_{3}(X)=I_{3} X \quad \text { avec } \quad I_{3}=\text { matrice identité d'ordre } 3 .
$$

On calcule alors le découplage pour le système (10), c'est-à-dire les matrices $\Delta(X)$ et $\Delta_{0}(X)$. On obtient les conditions de valıdité du découplage pour $\operatorname{Det}[\Delta]=a_{1} a_{2} b_{1} e_{\mathrm{c}} x_{3}^{2} \neq 0$ qui sont données par :

$$
e_{\mathrm{c}} \neq 0 \quad \text { et } \quad x_{3} \neq 0 \text {. }
$$

La condition sur la tension d'entrée n'est pas la plus gênante puisque qu'elle sera généralement vérifiée en fonctionnement normal. Par contre, nous étudierons de façon détaillée le démarrage du système et le fonctionnement à faible courant.

Le calcul du découplage conduit à des degrés relatifs égaux à $r_{1}=r_{2}=r_{3}=1$ donc le système se trouve complètement découplé $\left(r_{1}+r_{2}+r_{3}=3=n\right)$ et chaque sortie peut être représentée par un intégrateur.

Sous les conditions exprimées dans (30), les valeurs obtenues pour le calcul du découplage sont données par (31).

$$
\Delta_{0}(X)=\left(\begin{array}{c}
0 \\
0 \\
-b_{0} x_{3}
\end{array}\right), \Delta(X)^{-1}=\left(\begin{array}{ccc}
\frac{x_{1}-e_{\mathrm{c}}}{a_{1} e_{\mathrm{c}} x_{3}} & \frac{x_{2}-e_{\mathrm{c}}}{a_{2} e_{\mathrm{c}} x_{3}} & \frac{1}{b_{1} e_{\mathrm{c}}} \\
\frac{x_{1}}{a_{1} e_{\mathrm{c}} x_{3}} & \frac{x_{2}-e_{\mathrm{c}}}{a_{2} e_{\mathrm{c}} x_{3}} & \frac{1}{b_{1} e_{\mathrm{c}}} \\
\frac{x_{1}}{a_{1} e_{\mathrm{c}} x_{3}} & \frac{x_{2}}{a_{2} e_{\mathrm{c}} x_{3}} & \frac{1}{b_{1} e_{\mathrm{c}}}
\end{array}\right) .
$$

Le retour d'état peut donc s'exprimer par :

$$
\alpha(X)=-\Delta^{-1}(X) \Delta_{0}(X)=\left(\begin{array}{c}
\frac{-b_{0} x_{3}}{b_{1} e_{\mathrm{c}}} \\
\frac{-b_{0} x_{3}}{b_{1} e_{\mathrm{c}}} \\
\frac{-b_{0} x_{3}}{b_{1} e_{c}}
\end{array}\right) \text { et } \quad \beta(X)=\Delta^{-1}(X) .
$$


En appliquant sur le système le retour d'état, on obtient le système linéarisé suivant :

$$
\left\{\begin{array}{l}
\dot{y_{1}}=v_{\mathrm{c}_{1}}=v_{1} \\
\dot{y_{2}}=v_{\mathrm{c}_{2}}=v_{2} \\
\dot{y_{3}}=\dot{i_{\ell}}=v_{3} .
\end{array}\right.
$$

Avant de présenter les résultats de simulation, nous allons présenter comment résoudre le problème des singularités.

Traitements des singularités. - Comme nous l'avons remarqué lors du calcul du découplage pour un hacheur 3 cellules, nous obtenons deux singularités qui sont données par $x_{3}=i_{\ell}=0$ et $e_{\mathrm{c}}=0$ Pour ces deux points de fonctionnement et dans leurs voisinages respectifs, la commande par découplage non linéaire présentée perd sa validité. Il nous faut donc d'abord au niveau de la régulation détecter ces cas et leur proximité et ensuite envisager pour ces cas particuliers une régulation qui nous permette de conserver le contrôle du système.

- Passage par zéro de la tension d'entrée $e_{\mathrm{c}}$.

La première observation est que le passage par zéro de la tension d'entrée sera beaucoup plus simple à traiter que le passage par zéro du courant de charge. En effet, en fonctionnement normal, la tension d'entrée $e_{\mathrm{c}}$ sera toujours égale ou supérieure à une tension donnée positive.

Pour éviter cette singularité, nous bloquerons la commande tant que ou dès que la tension d'entrée sera inférieure à une tension critique donnée. Pour un hacheur $p$ cellules, les tensions internes des condensateurs en régime permanent correspondent à des fractions de la tension d'entrée égales à :

$$
v_{\mathrm{c}_{k}}=\frac{k e_{\mathrm{c}}}{p}
$$

On prendra donc une tension minimale d'entrée de fonctionnement $e_{\mathrm{c}_{\operatorname{man}}}$ inférieure à $\frac{e_{\mathrm{c}}}{p}$. Donc tant que l'on aura $e_{\mathrm{c}} \leq e_{\mathrm{c}_{\mathrm{rm}} \text { n }}$, la commande par découplage non linéaire ne sera pas opérationnelle et le problème de singularité par rapport à la tension d'entrée sera alors résolu.

- Passage par zéro du courant $x_{3}=i_{\ell}$

Nous avons précédemment montré que lors du passage par zéro du courant de charge $\left(x_{3}=0\right)$, la procédure de découplage conduisait à une singularité.

Détecter le passage par zéro dans notre procédure de régulation ne pose pas de problème particulier. De ce test découle le traitement suivant :

$-x_{3} \neq 0$ : on régule alors en utilisant la procédure de découplage,

$-x_{3}=0$ : on passe en franchissement de singularité.

Par l'observation de la structure du système, on peut aisément en déduire que lorsque le courant est nul, ou au voisinage de zéro, l'influence des commandes appliquées en valeur moyenne pour la commande des tensions est très faible, voire nulle. Dans ce cas, quelle que soit la différence de rapport cyclique moyen appliquée aux bornes d'un condensateur, la valeur de la tension à ses bornes n'évoluera que très peu.

La deuxième observation nous montre que lorsque le courant est nul (au démarrage par exemple), notre action peut être une commande simple de type proportionnel afin de démarrer l'évolution du courant. 


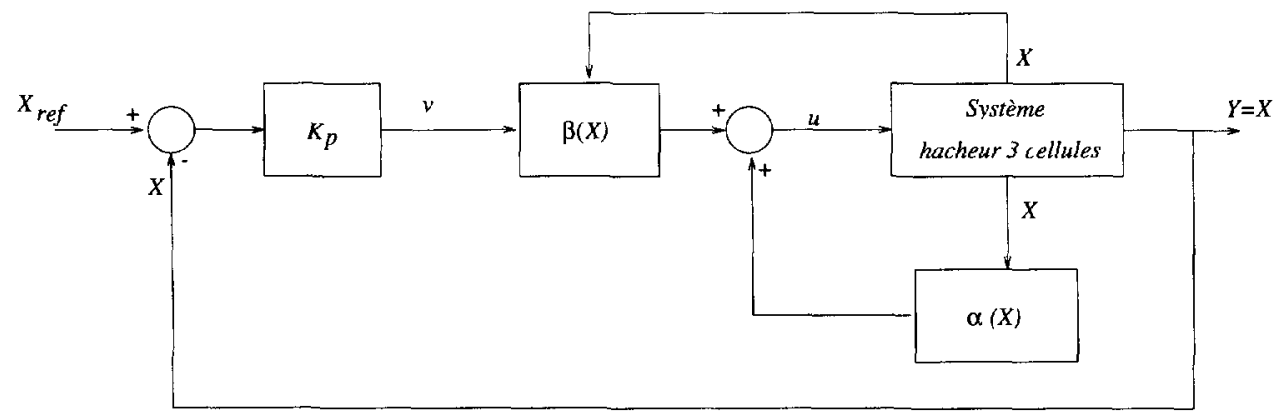

Fig 7. - Boucle de régulation avec correcteur proportionnel

[Proportionnal control loop]

Ainsi, notre procédure de traitement $\left(x_{3}=0\right)$ se résumera donc à effectuer une régulation proportionnelle sur chaque variable d'état.

Nous avons donc montré que pour effectuer une régulation par découplage, deux tests, l'un sur la variable d'état $x_{3}$ et l'autre sur $e_{\mathrm{c}}$ étaient à effectuer afin de traiter les cas singuliers dus à la structure du montage.

Il est tout de même intéressant de noter que la singularité $x_{3}=0$ vient du fait qu'avec le modèle aux valeurs moyennes, lorsque le courant est nul les tensions condensateurs sont des variables d'état non commandables. Ceci explique pourquoi la procédure de découplage n'est plus valable. On remarque donc que c'est le fait de vouloir découpler les tensions internes $v_{\mathrm{c}_{1}}$ et $v_{\mathrm{c}_{2}}$ par rapport à $i_{\ell}$ qui induit cette singularité.

3.2.2. Boucle de régulation proportionnelle $(P)$. - Après le retour d'état non linéaire, nous obtenons donc trois sous-systèmes découplés, représentés par des intégrateurs (Fig. 6). Nous allons mettre en place des correcteurs linéaires afin de réguler les trois grandeurs d'état.

Pour chacune des variables d'état, nous allons envisager tout d'abord un correcteur proportionnel dont l'équation pour la commande de la boucle $k$ est donnée par (35). La figure 7 représente la boucle de régulation linéaire appliquée au système découplé.

$$
v_{k}=K_{p_{k}}\left(x_{k_{\text {ref }}}-x_{k}\right) \text { avec } 1 \leq k \leq m \text { et } K_{p_{k}}>0
$$

On obtient alors pour chaque variable d'état une fonction de transfert en boucle ouverte (BO) du type :

$$
T_{B O_{k}}(p)=\frac{K_{p_{h}}}{p}
$$

et donc en boucle fermée :

$$
T_{B F_{h}}(p)=\frac{1}{1+\tau_{k} p} \quad \text { avec } \quad \tau_{k}=\frac{1}{K_{p_{k}}} .
$$

On imposera donc sur chaque boucle la dynamique désirée. Dans ces conditions, nous pouvons avec ce type de régulateur imposer.

- une dynamique donnée pour chaque variable d'état, 

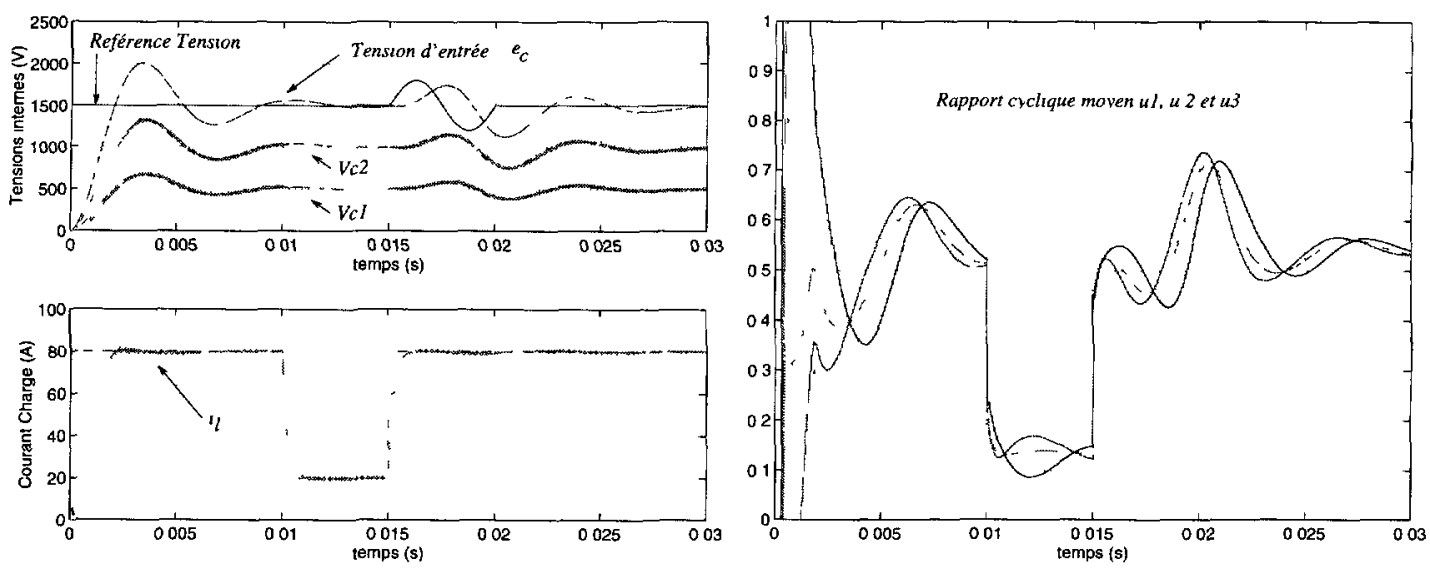

(A)

(B)

Fig. 8. - Simulation avec découplage.

[Simulation with input/output linearization]

- une erreur statique nulle en régime permanent, ceci étant dû au fait que les boucles possèdent un intégrateur dans la chaine.

- Validation du découplage

Le but de cette première simulation (Fig. 8) est de montrer le fonctionnement de la procédure de découplage. Les paramètres utilisés sont alors :

$$
\begin{aligned}
& R=10 \Omega \\
& L=1,5 \mathrm{mH} \\
& K_{p 1}=K_{p 2}=K_{p 3}=5000 \\
& T_{\mathrm{e}}=62,5 \mu \mathrm{s} \\
& C_{1}=C_{2}=40 \mu \mathrm{F} \\
& r_{\mathrm{e}}=0,6 \Omega \\
& L_{\mathrm{e}}=1 \mathrm{mH} \\
& C_{\mathrm{e}}=500 \mu \mathrm{F} .
\end{aligned}
$$

Le cycle imposé aux 3 variables d'état est le suivant :

$$
\begin{array}{ll}
t=0 & : \text { démarrage du courant } i_{\text {ref }}=80 \mathrm{~A} \\
t=10 \mathrm{~ms} & : \text { variation de }-60 \mathrm{~A} \text { de } i_{\text {ref }} \\
t=15 \mathrm{~ms} & : \text { variation de }+60 \mathrm{~A} \text { de } i_{\text {ref }} \\
t=15 \mathrm{~ms} & : \text { perturbation sinusoïdale de } e_{\mathrm{c}} \text { (amplitude } 300 \mathrm{~V} \text { ). }
\end{array}
$$

Le calcul des dynamiques imposées aux variables d'état (37) a été fait pour ne pas saturer les commandes $u_{k}$ qui représentent les rapports cycliques moyens et qui restent compris dans l'intervalle $[0 \ldots 1]$. On note donc sur la figure 8 un bon découplage de toutes les grandeurs d'état.

On remarque que les variations imposées sur les boucles de tension internes $\left(v_{c_{1 \text { ref }}}\right.$ et $\left.v_{c_{2 \text { ref }}}\right)$ dépendent de la constante de temps du filtre d'entrée puisque que l'on a exactement $v_{\mathrm{c}_{1 \text { ref }}}=e_{\mathrm{c}} / 3$ et $v_{\mathrm{c}_{\text {ref }}}=2 e_{\mathrm{c}} / 3$. 

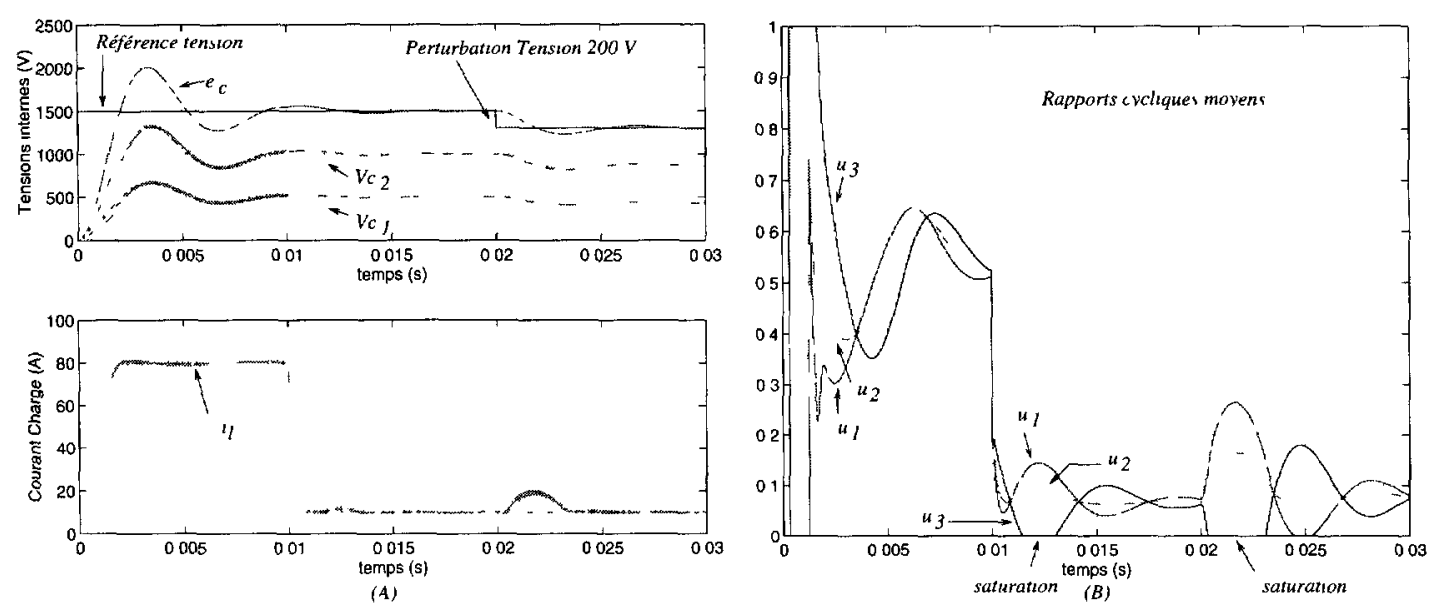

Fig. 9 - Saturation des rapports cyclıques à faible charge.

[Duty cycle saturation with small load current.]

\section{- Mise en évidence des saturations des rapports cyclıques}

$\mathrm{Au}$ cours de cette deuxième simulation, nous allons montrer le problème de saturation des rapports cycliques lorsque le courant dans la charge est faible. Les paramètres de simulation sont les mêmes que (38).

Le cycle imposé est cette fois le suivant :

$$
\begin{array}{ll}
t=0 & : \text { démarrage du courant } \imath_{\mathrm{ref}}=80 \mathrm{~A} \\
t=10 \mathrm{~ms} & : \text { variation de }-70 \mathrm{~A} \text { de } i_{\text {ref }} \\
t=20 \mathrm{~ms} & : \text { variation de }-200 \mathrm{~V} \text { de } e_{\mathrm{c}_{\text {ref }}} .
\end{array}
$$

Sur la figure 9, la simulation nous montre que le fait d'imposer une variation des tensions condensateurs à faible courant de charge $(t=20 \mathrm{~ms})$ entraîne une perturbation du courant $i_{\ell}$ due à la saturation de la commande $u_{3}$ que l'on remarque sur la figure $9 \mathrm{~B}$.

3.2.3. Étude du problème de saturation des commandes - Comme nous le montre la simulation précédente, les saturations des commandes $u_{k}$ posent des problèmes et nécessitent une étude particulière Nous devons en effet étudier le cas où la commande fixe des entrées $u_{k}$ hors de l'intervalle $[0 \ldots 1]$.

Diverses solutions sont alors envisageables et les plus simples consistent à limiter soit les dynamiques de réglage $\left(K_{p_{k}}\right)$, soit la dynamique d'évolution de la tension d'entrée $e_{\mathrm{c}}$ (en jouant sur les paramètres du filtre d'entrée). Nous étudierons pour notre part une procédure de gestion des saturations afin de limiter leurs effets.

On pose d'abord comme hypothèse d'étude que les trois rapports cycliques seront toujours ordonnés comme le montre la figure 10. Cette hypothèse n'est pas trop restrictive car nous imposerons le plus souvent pour les deux tensions $v_{c_{1}}$ et $v_{c_{2}}$ des varıations de même signe donc des différences $\delta_{1}=u_{1}-u_{2}$ et $\delta_{2}=u_{2}-u_{3}$ de même signe Les différents cas avec $\operatorname{sign}\left(\delta_{1}\right) \neq \operatorname{sign}\left(\delta_{2}\right)$ ne seront pas étudiés car ils correspondent à des situations où de fortes dynamiques ne sont pas nécessaires. 


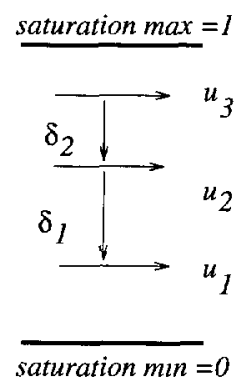

CAS I

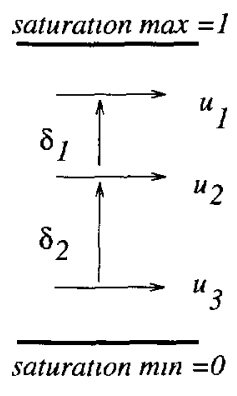

CAS 2

Fig. 10. - Hypothèse d'étude

[Study proposition.]

On rappelle que le modèle peut s'exprimer sous la forme suivante :

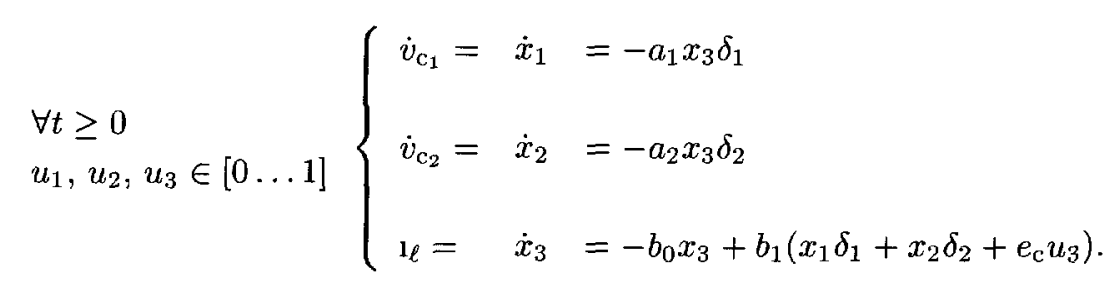

La procédure de découplage impose $u=\alpha(X)+\beta(X) v$ et la structure du système impose des saturations $u_{\mathrm{s} k}=\max \left(\min \left(u_{k}, 1\right), 0\right)$.

La figure 11 nous montre différents cas possibles de saturation sous les hypothèses fixées précédemment. Les cas $(1 \mathrm{~A})$ et $(1 \mathrm{~B})$ correspondent à des $\delta_{k}$ négatifs alors que les cas $2 \mathrm{~A}$ et $2 \mathrm{~B}$ correspondent à des $\delta_{k}$ positifs.

Les cas $1 \mathrm{~B}$ et $2 \mathrm{~A}$ sont symétriques et correspondent chacun à un cas de figure où une au moins des tensions condensateurs voit son évolution bloquée car $\delta_{1}=0$ après saturation. Pour ces deux cas nous effectuerons une homothétie de $u_{1}$ et $u_{2}$ en gardant $u_{3}$ et le rapport $\frac{\delta_{1}}{\delta_{2}}$ constant afin d'avoir $\delta_{\perp} \neq 0$ et $\delta_{2} \neq 0$, c'est-à-dire une dynamique de commande non nulle pour toutes les tensions internes.

Les cas $1 \mathrm{~A}$ et $2 \mathrm{~B}$ sont également symétriques et représentent une saturation de la commande $u_{3}$. Dans ce cas on observe principalement une perturbation du courant et une perturbation de $v_{\mathrm{c}_{2}}\left(\delta_{2}\right.$ saturé). Ces deux cas seront traités de la façon suivante (cas $2 \mathrm{~B}$ ) $\cdot$ on gardera constante la valeur $\left(x_{1} \delta_{1}+x_{2} \delta_{2}+e_{\mathrm{c}} u_{3}\right)$ de l'équation (39) afin de supprimer la perturbation du courant en posant $u_{3 \mathrm{~s}}=0$ et $\delta_{1}=\sigma \delta_{2}$.

On aura donc :

$$
\left\{\begin{array}{cl}
x_{1} \delta_{1}+x_{2} \delta_{2}+e_{\mathrm{c}} u_{3} & =x_{1} \sigma \delta_{2 s}+x_{2} \delta_{2 s}+e_{\mathrm{c}} u_{3 s} \\
\delta_{1} & =\sigma \delta_{2} .
\end{array}\right.
$$


CAS l A

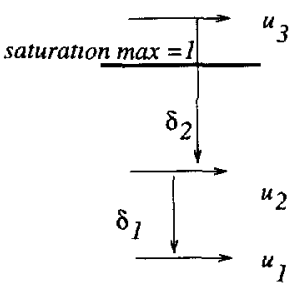

$\overline{\text { saturation } \min }=0$

CAS 2 A

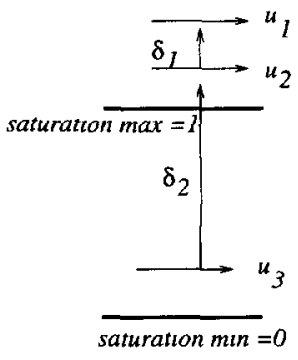

CAS $1 \mathrm{~B}$

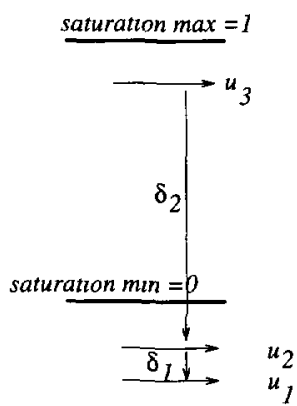

CAS 2 B

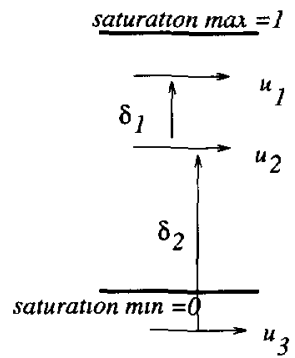

Fig. 11 - Cas d'étude de la saturation.

[The different cases of saturation.]

De l'équation (40), on tire les nouvelles valeurs de $\delta_{1 \mathrm{~s}}$ et $\delta_{2 \mathrm{~s}}$ de la façon suivante :

$$
\left\{\begin{array}{l}
\delta_{2 \mathrm{~s}}=\frac{x_{1} \delta_{1}+x_{2} \delta_{2}+e_{\mathrm{c}} u_{3}}{x_{1} \sigma+x_{2}} \\
\delta_{1 \mathrm{~s}}=\sigma \delta_{2 \mathrm{~s}} .
\end{array}\right.
$$

Le traitement du cas $1 \mathrm{~A}$ s'effectuera de la même façon en posant $u_{3 \mathrm{~s}}=1$.

La figure 12 nous montre la même simulation qu'en 9 avec cette fois le traitement actif des saturations. On remarque que le courant n'est cette fois plus perturbé par les saturations des commandes lors de la variation de la tension d'entrée $e_{\mathrm{c}}$. On remarque que le prix à payer pour avoir ce résultat est une limitation importante des dynamıques des tensions internes $(t=20 \mathrm{~ms})$ obtenues par le re-calcul de $\delta_{1}$ et $\delta_{2}$ de l'équation (41).

En effet, un découplage exact du courant impose des dynamiques de variations pour les tensions très faibles et provoque un déséquilibre sur les tensions aux bornes des interrupteurs, normalement égales à $v_{\mathrm{T}_{2}}=e_{\mathrm{c}} / 3$ en régime permanent. Pour limiter ce déséquilibre, nous devons introduire une priorité soit à un courant découplé, soit à un déséquilibre réduit. La procédure pour introduire une priorité sera la suivante : si une tension $v_{\mathrm{Tr}_{2}}=v_{\mathrm{T} 2}-e_{\mathrm{c}} / 3$ dépasse un seuil de sécurité fixé $\left(V_{\text {seuil }}=0,5 e_{\mathrm{c}} / 3\right)$, on ne traitera pas la saturation pour forcer le rééquilibrage des tensions en admettant une perturbation du courant. La figure 13 nous montre le cas où le traitement de la saturation engendre à $t=22 \mathrm{~ms}$ un dépassement du seuil 

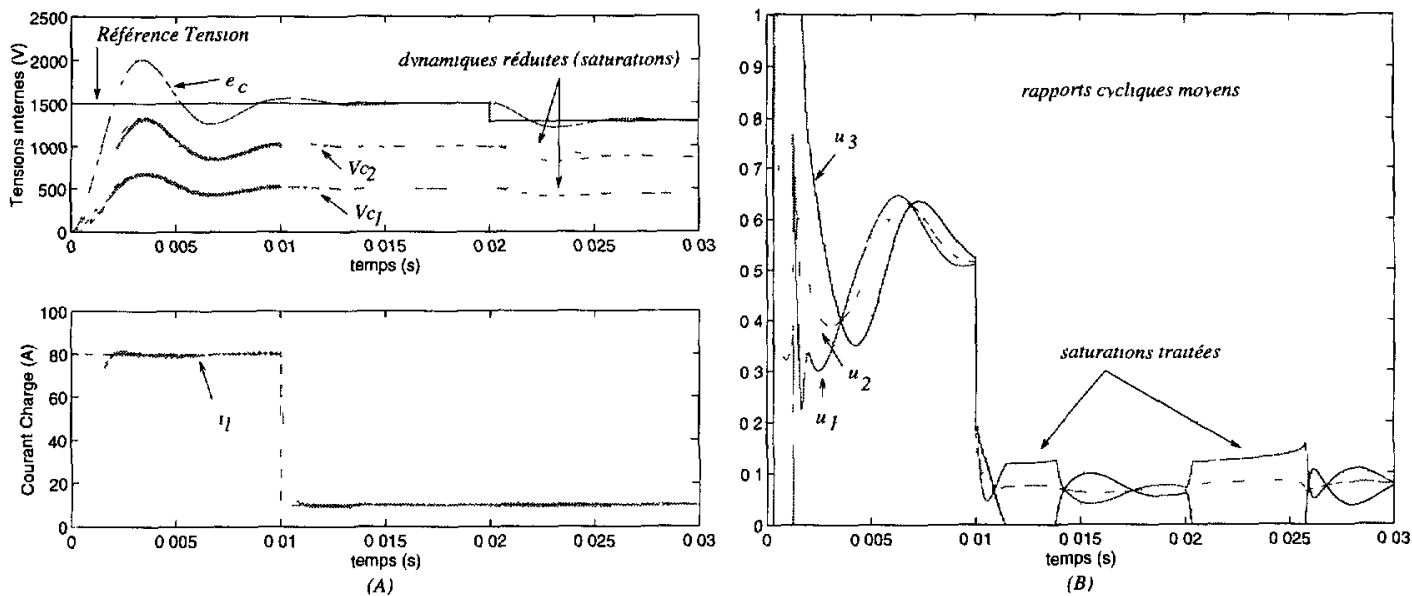

$(B)$

Fig 12. - Traitement des saturations . prorité au courant.

[Saturation control: load current priority.]
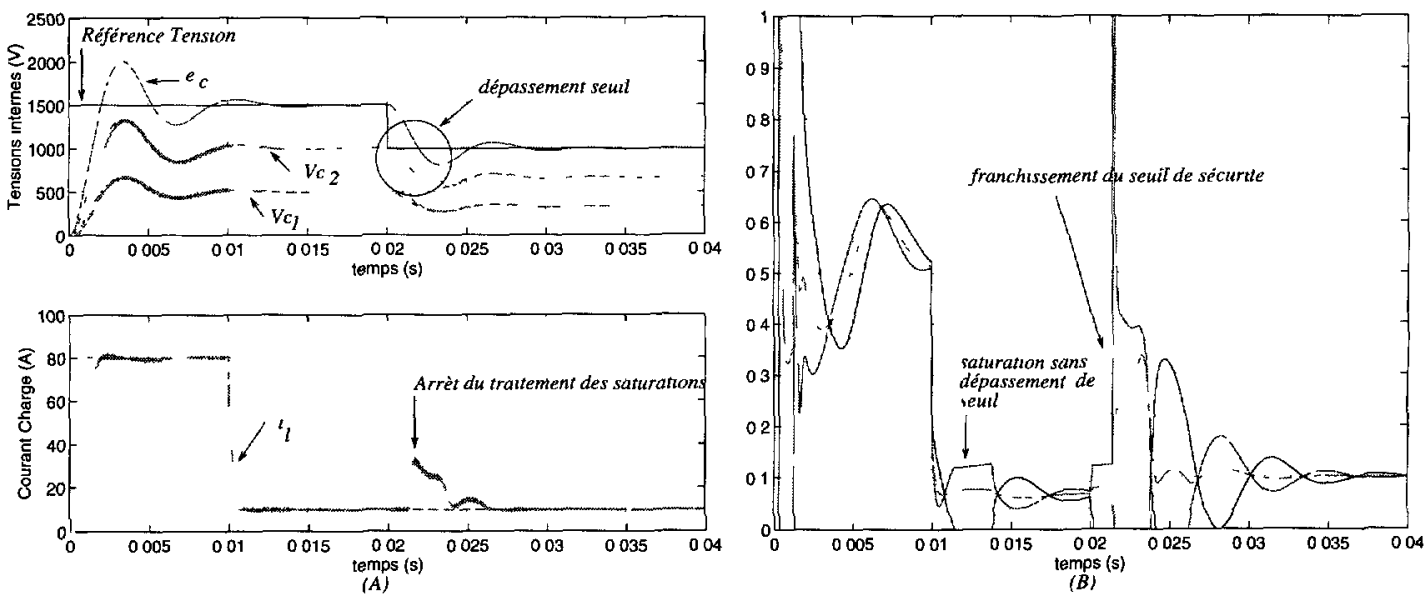

Fig 13. - Traitement des saturations : en cas de danger, priorité aux tensions.

[Saturation control in case of danger, capacitor voltage priority.]

de sécurité (Fig. 14) dû à un échelon de la tension de référence de $-500 \mathrm{~V}$ (au lieu de $-200 \mathrm{~V}$ ) à $t=20 \mathrm{~ms}$. Après un dépassement du seuil de sécurité, on ne traite plus la saturation afin de redonner la priorité à un rééquilibrage des tensions.

Le choix de ce seuil n'est pas unique et peut être fait par rapport à une tension relative à $e_{c} / 3$ (exemple choisi) ou en absolu en prenant en compte les contraintes maximum applicables sur les interrupteurs. On peut donc conclure que le traitement des saturations associé à un seuil de sécurité permet d'obtenir un bon compromis entre le découplage du courant et la régulation des tensions internes. 


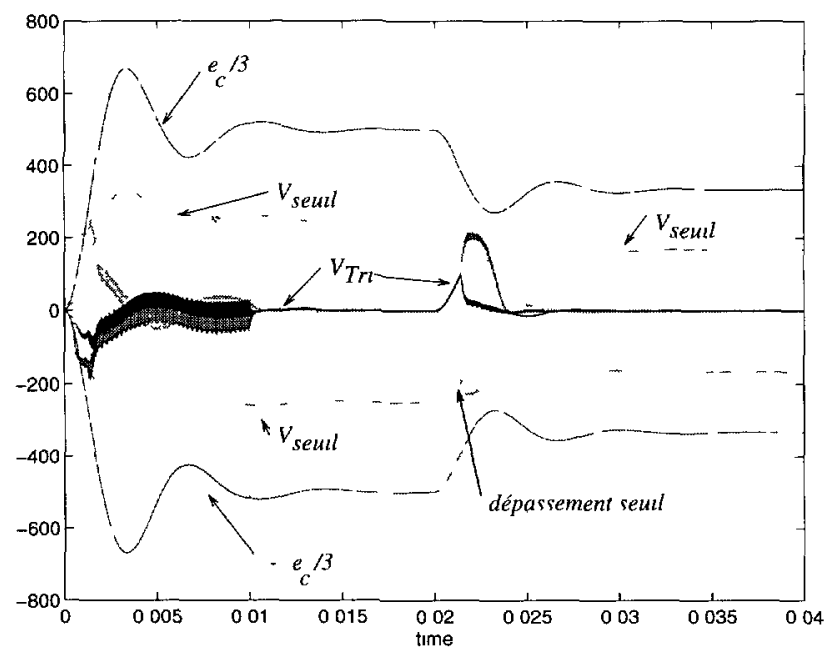

Fig. 14 - Dépassement du seuil de sécurité.

[Security level crossing.]

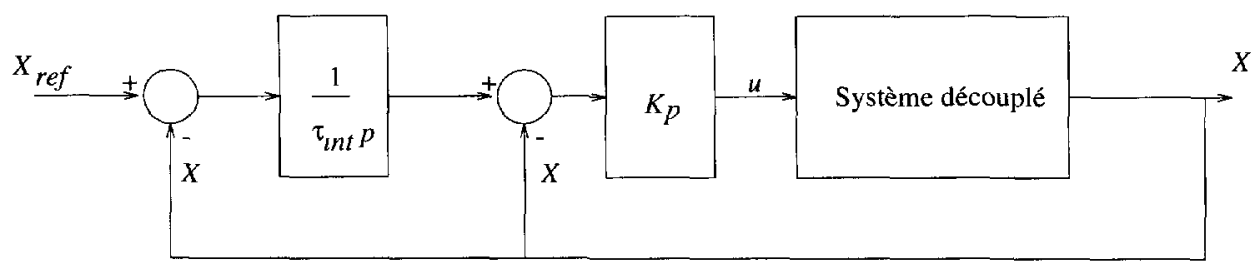

Fig 15. - Boucle avec correcteur IP

[Integral proportionnal loop.]

3.2.4. Boucle de régulation Intégrale Proportionnelle $(I P)$. - Nous avons vu au cours du paragraphe précédent qu'un correcteur proportionnel, bien que possédant une structure intéressante de par sa simplicité ne suffisait pas pour rejeter l'effet des perturbations qui pouvaient apparaître sur le système. Nous allons maintenant envisager une structure de régulation possédant une intégration. Cette structure devra annuler les perturbations sur le système comme par exemple l'apparition d'un pôle dans la fonction de transfert du système linéarisé due à une erreur de paramètres ou une perturbation sur les rapports cycliques moyens.

Plusieurs solutions s'offrent à nous. Soit prendre un correcteur de type PI ou IP, soit un correcteur plus complexe. Nous avons choisi d'implanter un correcteur de type IP qui possède deux boucles imbriquées comme nous le montre la figure 15 .

Le choix réside dans le fait que la dynamique imposée par le concepteur n'est pas modifiée par l'ajout d'un zéro dans la fonction de transfert en boucle ouverte comme on peut l'observer pour une structure PI classique [10]. La première boucle proportionnelle sera donc calculée comme précédemment, c'est-à-dire afin d'imposer une dynamique désirée sur chaque variable d'état. La deuxième boucle sera implantée pour compenser d'éventuelles perturbations. L'effet intégral du régulateur IP sera calculé de façon classique, c'est-à-dire afin d'assurer une bande passante et un amortissement donné. 

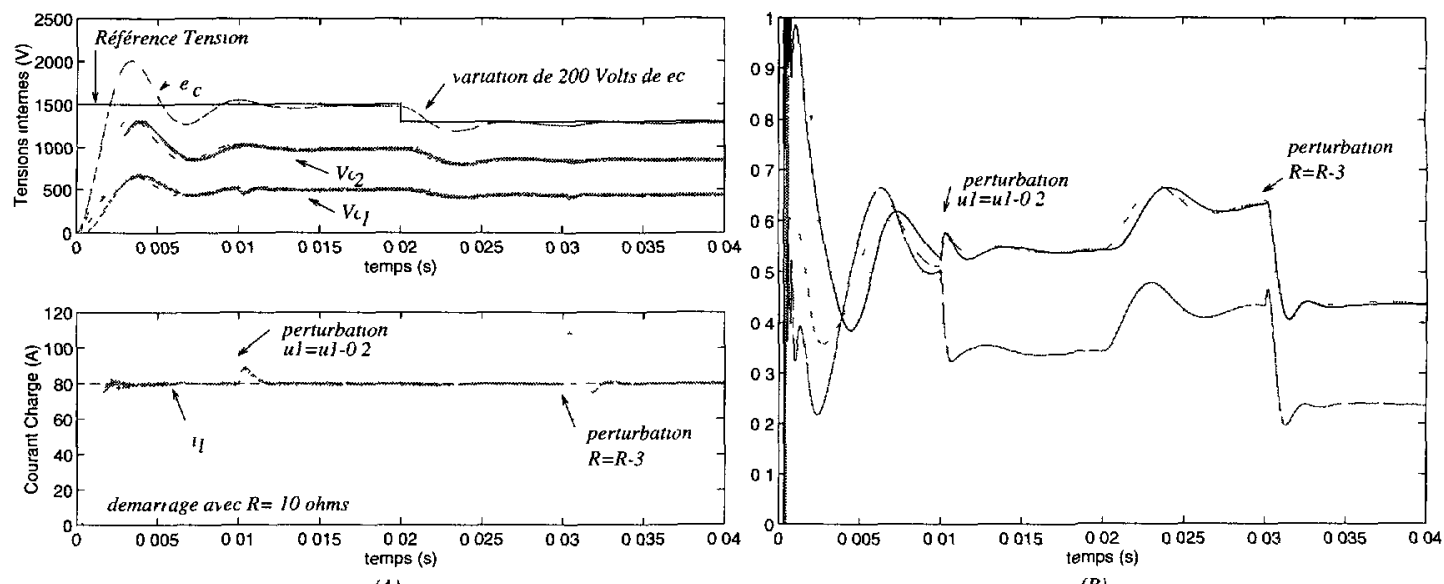

(A)

(B)

Fig 16. - Régulation de type IP.

[Simulation results with IP control.]

Le calcul du coefficient intégral s'effectuera dans le cas où le système est parfaitement découplé, donc à partir des équations données par (33).

Dans ce cas, la fonction de transfert en boucle fermée du système avec correcteur IP peut s'écrire :

$$
T_{\mathrm{BF}_{h}}(p)=\frac{1}{\tau_{k} \tau_{\mathrm{Int}_{k}} p^{2}+\tau_{\mathrm{Int}_{k}} p+1}=\frac{1}{r}=\frac{p^{2}}{\frac{2 m_{k}}{\omega_{\mathbf{0}_{k}}^{2}}+\frac{2}{\omega_{0_{h}}} p+1}
$$

avec $\tau_{k}=\frac{1}{K_{p_{k}}}$

On identifie alors les deux termes de l'égalité précédente afin d'exprimer $m_{k}$ et $\omega_{0_{k}}$ en fonction des paramètres $\tau_{k}$ et $\tau_{\mathrm{int}_{h}}$. On obtient:

$$
\left\{\begin{array}{l}
m_{k}=\frac{1}{2} \tau_{k} \omega_{0_{k}} \\
\omega_{0_{k}}=\sqrt{\frac{K_{p_{k}}}{\tau_{\mathrm{nnt}_{k}}}}
\end{array}\right.
$$

Par le choix de $K_{p_{h}}$ et $\tau_{\text {ntt }_{h}}$, on pourra donc fixer la bande passante du système et son amortissement. On effectuera d'abord le calcul des $K_{p_{k}}$ afin d'appliquer la dynamique désirée sur le système découplé, puis on calculera l'effet intégral afin d'obtenir $m_{k}$ et $\omega_{0_{k}}$ souhaités qui ne sont pas indépendants avec un IP dans ce cas.

Les figures $16 \mathrm{~A}$ et $16 \mathrm{~B}$ représentent un essai où pour chaque boucle nous avons pris $K_{p_{h}}=5000$ et $\tau_{\text {nt }_{k}}=550 \mu$ s. Les résultats nous montrent un bon comportement du système aussi bien pendant la phase de démarrage que lors de l'apparition des perturbations. La première perturbation ( $t=10 \mathrm{~ms}$ ) consiste en une variation de $u_{1}$ de $20 \%$ de sa valeur maximum alors que la deuxième perturbation $(t=30 \mathrm{~ms})$ est donnée par une variation de la résistance de charge de $3 \Omega$. 

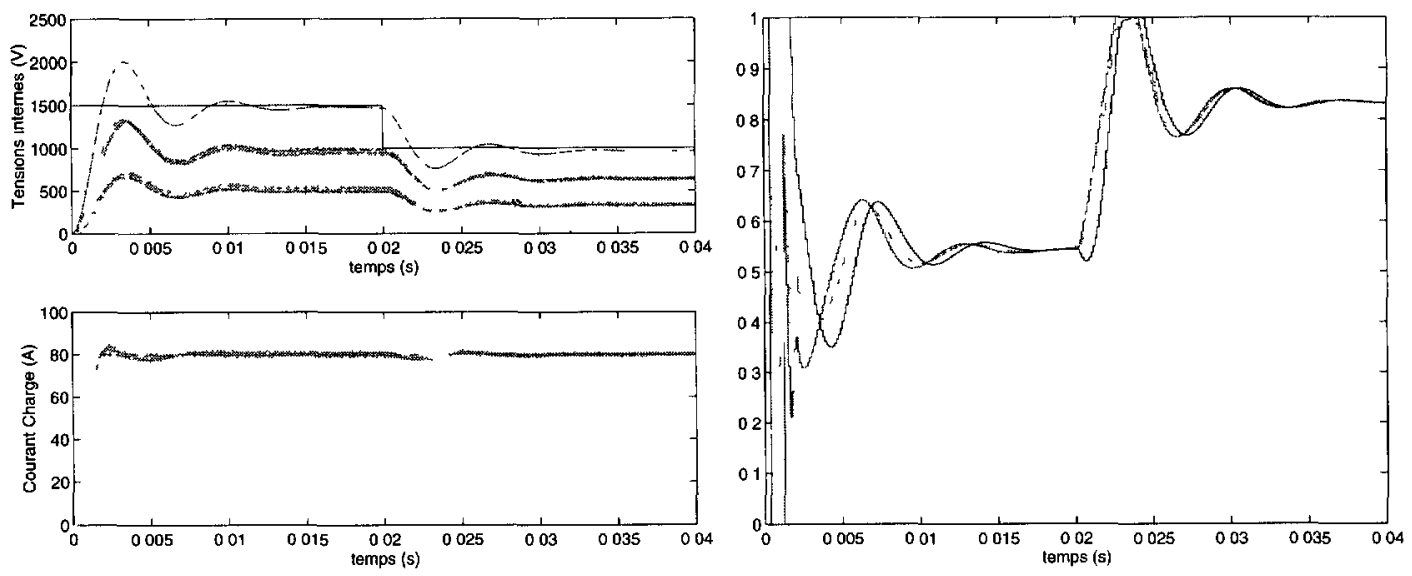

(A) Te=125 micro-secondes


(B) $T_{e}=1875$ micro secondes

Fig. 17 - Influence de la pérıde d'échantillonnage

[Sampling period effects ]

\subsection{Problèmes d'implantation numérique de la commande}

3.3.1. Période d'échantıllonnage. - Les résultats précédemment présentés ont été obtenus avec une période d'échantillonnage $T_{\mathrm{e}}=62,5 \mu \mathrm{s}$ ce qui correspond à la prise d'un échantillon par période de découpage. La valeur optimale de cette période est relativement difficile à déterminer puisqu'elle dépend évidemment des dynamiques du système donc de la charge et de la valeur des capacités internes. Avec les valeurs de composants utilisés, nous avons obtenu pour $T_{\mathrm{e}}=62,5 \mu \mathrm{s}$ de bons résultats. Les figures suivantes $17 \mathrm{~A}$ et $17 \mathrm{~B}$ nous montrent deux essais avec respectivement $T_{\mathrm{e}}=125 \mu \mathrm{s}$ et $T_{\mathrm{e}}=187,5 \mu \mathrm{s}$. Les résultats obtenus restent acceptables même si cette fois la qualité du découplage diminue.

3.3.2. Implantation numérique. - L'implantation sur calculateur numérique d'un algorithme de commande pose toujours des problèmes de temps de calcul. En effet, dans toutes les simulations présentées précédemment, nous avons supposé que le calcul de l'algorithme de commande était instantané. 


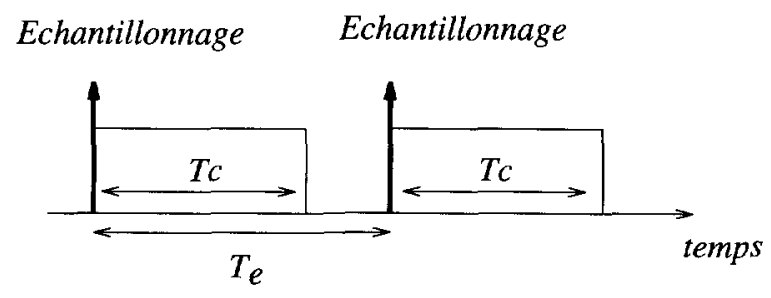

Tc=temps de calcul

Fig. 18 - Temps de calcul de la commande.

[Algorithm calculation time.]

La procédure de découplage pour un hacheur 3 cellules a été écrite en langage $\mathrm{C}$ puis exécutée sur un PC équipé d'un processeur PENTIUM $166 \mathrm{MHz}$. Nous avons obtenu un temps d'exécution global de $33 \mu$ s incluant les conversions $\mathrm{A} / \mathrm{N}$ et la programmation numérique des rapports cycliques.

La figure 18 représente le décalage entre l'acquisition effectuée par les convertisseurs $\mathrm{A} / \mathrm{N}$ et les commandes réellement envoyées aux convertisseurs. Ce décalage dépend essentiellement du temps de calcul et peut être représenté en simulation par un retard pur correspondant à $T_{\mathrm{e}}$. Après avoir intégré dans notre système de simulation ce temps de calcul, nous avons effectué deux simulations données par les figures $19 \mathrm{~A}$ et $19 \mathrm{~B}$. On note que la dégradation des résultats obtenus précédemment est beaucoup plus rapide que lorsqu'on augmentait simplement la période d'échantillonnage. En effet, le temps de calcul dans ce cas fait partie des dynamiques non modélisées par la représentation choisie du système et donc influe de façon importante sur le découplage. Les résultats obtenus avec une période de $62,5 \mu$ s et un temps de calcul de $62,5 \mu \mathrm{s}$ restent acceptables alors que pour $T_{\mathrm{e}}=125 \mu$ s et un temps de calcul de $62,5 \mu \mathrm{s}$, les résultats constituent un cas limite de fonctionnement.

Il serait alors intéressant de développer un prédicteur d'état afin de pouvoir compenser ce retard dû au temps de calcul de la commande. À chaque période $k T_{\mathrm{e}}$, on estime en fonction des commandes l'état futur à l'instant $(k+1) T_{\mathrm{e}} \widetilde{X}_{(k+1) T_{\mathrm{e}}}$. Il est important que ce prédicteur se base sur un modèle dont les paramètres sont à la fois bien identifiés et invariants dans le temps, sous peine de perdre l'intérêt de ce type de compensation.

\subsection{VALIDATION EXPÉRIMENTALE DE L'ALGORITHME DE RÉGULATION}

3.4.1. Présentation du système expérimental. - Afin de valider ces algorithmes de découplage, nous avons conçu, étudié et réalisé un système de commande numérique dédié aux convertisseurs multıcellulaires. La figure 20 représente le synoptique de cette réalisation. L'organe de calcul est un processeur PENTIUM $166 \mathrm{MHz}$ avec 32 Mo de RAM.

Nous avons développé une carte d'entrées/sorties numériques pour ce processeur dont les principales caractéristiques sont les suivantes :

48 entrées/sorties numériques,

6 convertisseurs analogique-numérique $\left(T_{\text {conversion }}=2 \mu \mathrm{s}\right)$

6 convertisseurs numérique-analogique $\left(T_{\text {conversion }}=1 \mu \mathrm{s}\right)$.

Le calcul de la commande s'effectue par un programme d'interruptions déclenché par un signal externe provenant de la carte LIAISON. La période de ce signal est de $62,5 \mu \mathrm{s}$. L'acquisition des grandeurs d'état s'effectue au travers de filtres de Butterworth d'ordre 4 situés sur la carte 

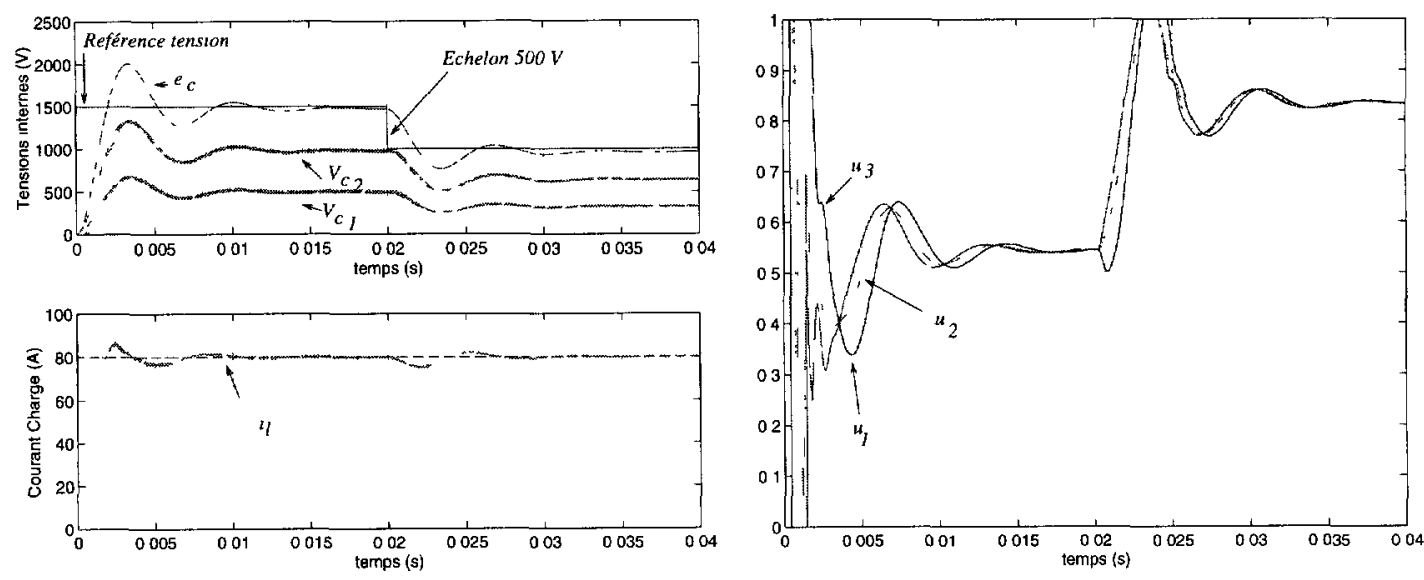

(A) Te=62 5 moro-secondes, et retard pur de Te
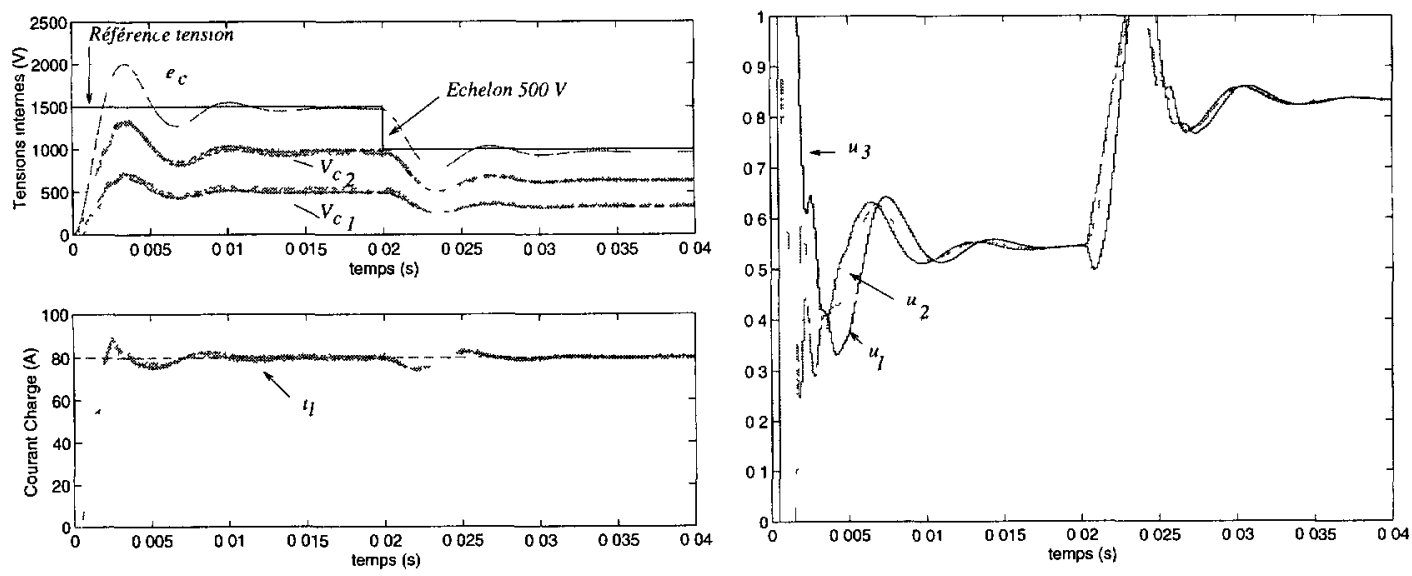

(B) $T e=125$ micro-secondes, et retard pur de 625 micro-secondes

Fig. 19. - Influence du temps de calcul de la commande.

[Algorithm computation time influence]

CAPTEURS. Les interrupteurs de chaque cellule de commutation sont pilotés par une carte indépendante permettant de fonctionner en rapport cyclique et phases variables. La structure permet de fonctionner avec un maximum de 256 cellules de commutation.

La figure 21 est une reproduction photographique du système de commande où l'on peut voir apparaître de gauche à droite la carte LIAISON permettant d'assurer les connexions avec le PC, les cartes CELLuLES pilotant directement les interrupteurs d'une cellule de commutation à l'aide de fibre optique et la carte CAPTEURs permettant le filtrage analogique et la mise en forme des mesures.

La figure 22 est une reproduction photographique des trois types de carte disponibles dans le système de commande avec de gauche à droite la carte LIAISON, la carte CELLULE et enfin la carte CAPTEURS.

La partie puissance étant encore en cours de développement, nous avons validé cette commande sur un simulateur analogique [14] de convertisseurs statiques permettant le fonctionnement en temps réel et représenté sur la figure 23 . 


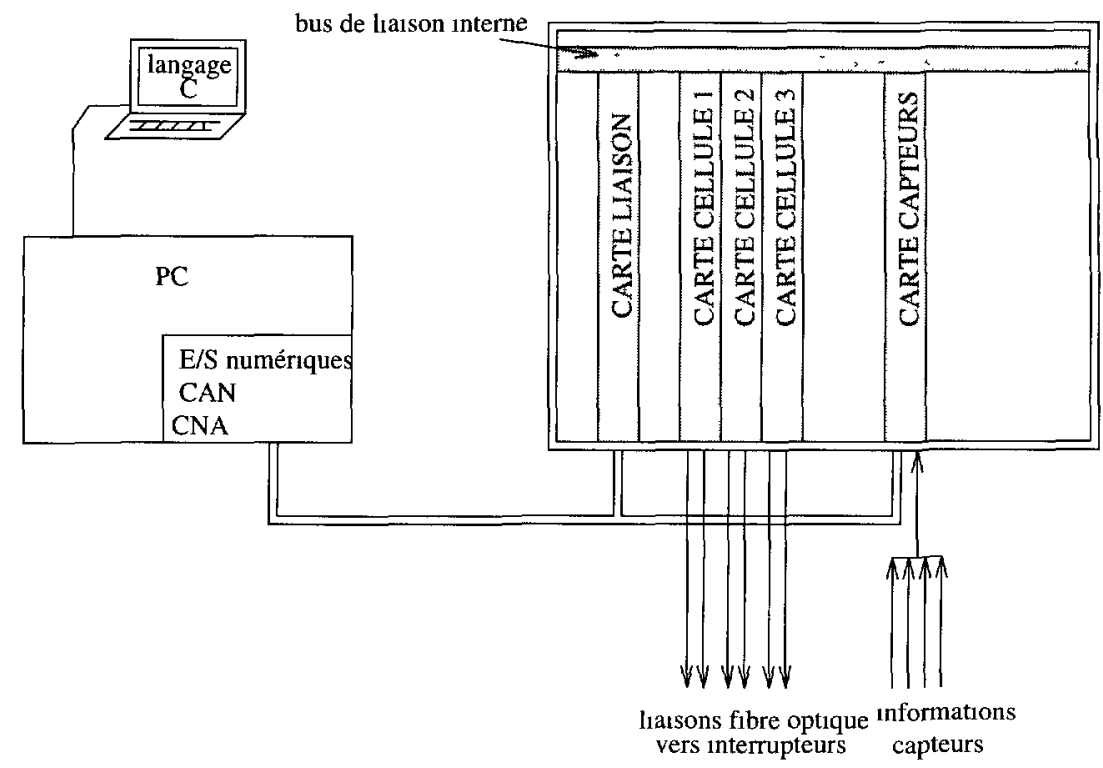

Fig. 20 - Synoptıque de la maquette numérique.

[Synoptic of the numerical realization]

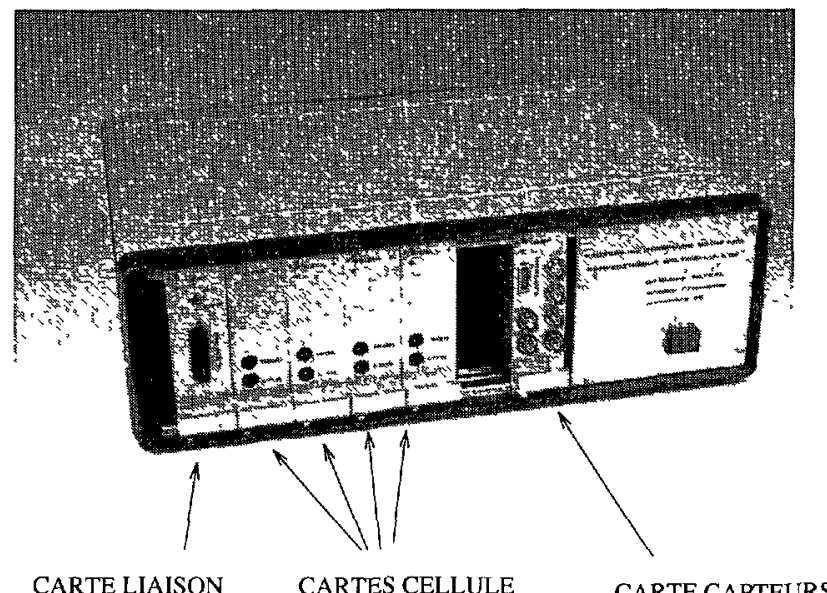

Fig. 21. - Système de commande numérique.

[Digital control system]

Ce simulateur nous offre la possibilité de fonctionner avec 3 cellules de commutation sur charge $R, L$ avec une fréquence de hachage de $16 \mathrm{kHz}$.

Cette première étape expérimentale est particulièrement intéressante pour nous, car elle nous permet de valider totalement l'algorithme de commande ainsi que le système de commande numérique complet et ce, en temps réel. L'étape suivante sera donc l'application de ces résultats sur la maquette réelle. 


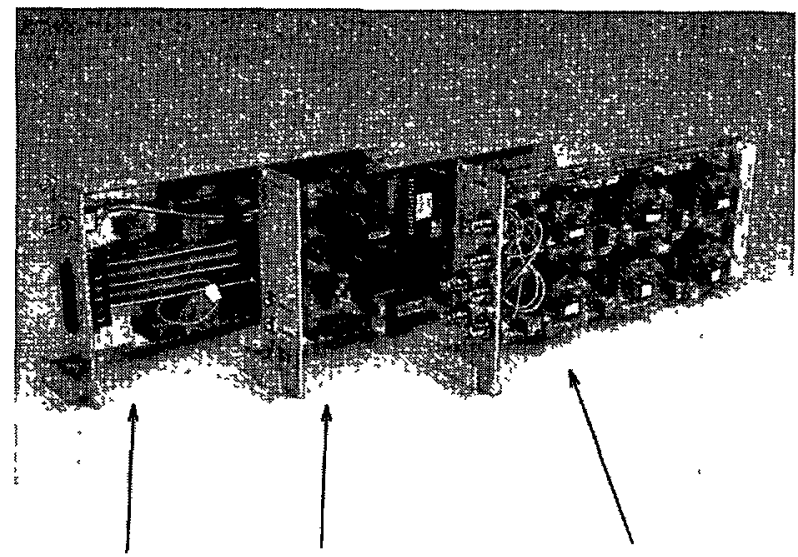

CARTE LiaISON CARTE CELLULE

CARTE CAPTEURS

Fig. 22 - Cartes de commande numérique

[Digital control boards.]

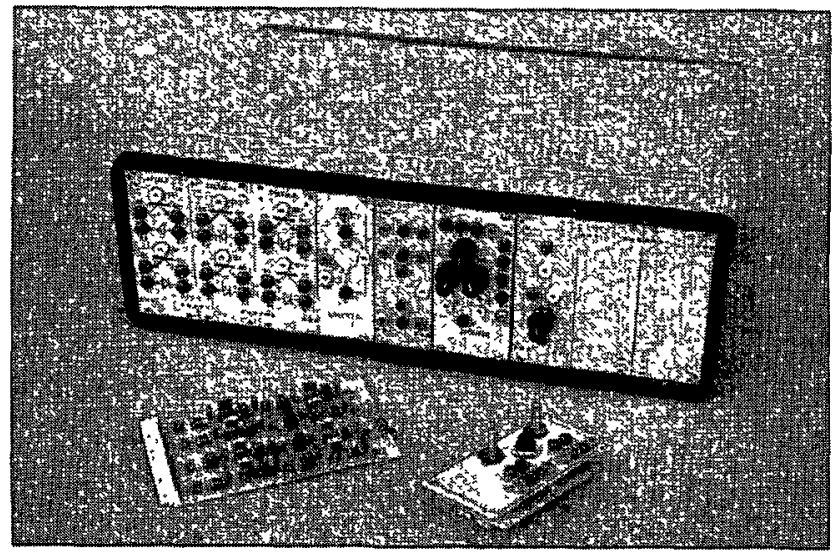

Fig. 23. - Simulateur analogique.

[Analog simulation system ]

3.4.2. Résultats expérimentaux. - Les essais expérimentaux ont été obtenus sur un hacheur 3 cellules représenté en figure 3 sans filtre d'entrée. Les paramètres du système sont ceux donnés dans l'équation (38).

Il est important de noter que le simulateur analogique est normalement conçu pour une fréquence maximum de $10 \mathrm{kHz}$. Dans notre cas, la fréquence de hachage est de $16 \mathrm{kHz}$ ce qui signifie que le système fonctionne au-delà de ses propres limites. Par exemple, le fonctionnement en boucle ouverte à cette fréquence n'est plus conforme au fonctionnement réel du système. Les principaux défauts apparaissant dans ce type d'utilisation du simulateur se situent au niveau des interrupteurs.

La figure 24 nous montre le fonctionnement en boucle fermée avec la régulation par découplage. Les tensions $v_{\mathrm{c}_{k}}$ sont bien asservies à $k e_{\mathrm{c}} / p$ et le courant de charge lors des transitoires 


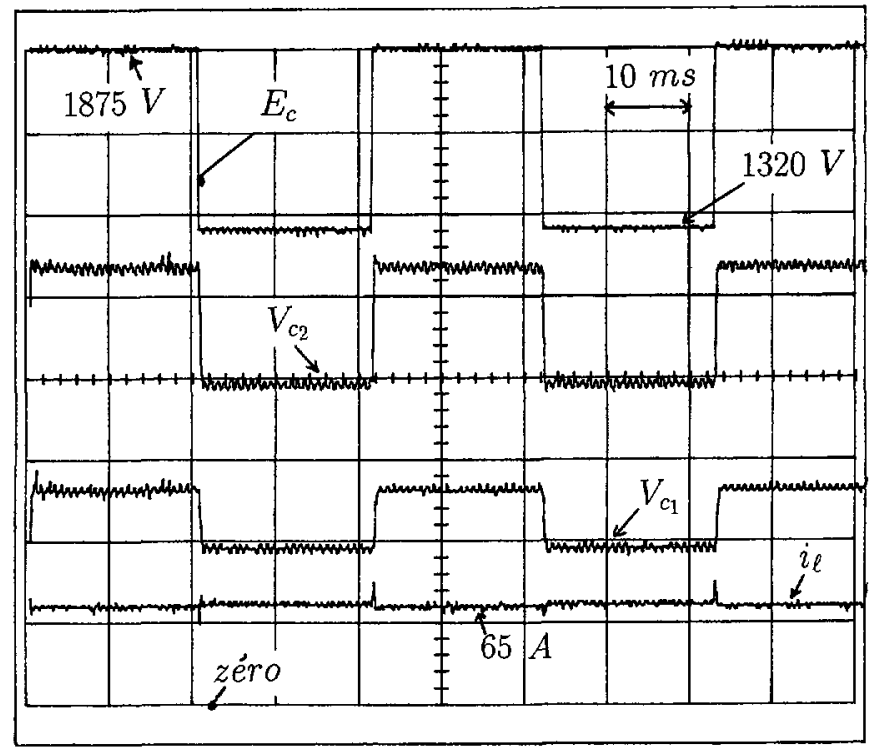

Fig. 24. - Chronogrammes des variables régulées.

[States variables closed loop waveforms.]



Fig. 25. - Chronogrammes des variables régulées (ZOOM).

[States variables closed loop waveforms (ZOOM).] 
de la tension d'entrée est faiblement perturbé. Cette perturbation est due au retard introduit par le calcul de la commande, comme les simulations nous l'avait déjà montré.

La figure 25 est un zoom de la figure précédente qui nous permet d'apprécier le temps de réponse des différentes variables d'état lors d'une variation de la tension d'entrée $e_{\mathrm{c}}$.

Ce type de régulation permet donc d'obtenir une bonne répartition des contraintes sur tous les interrupteurs quel que soit le régime de fonctionnement.

\section{Conclusions}

Au cours de notre étude, nous avons d'abord montré comment on pouvait à l'aide de l'exploitation de la structure du montage multicellulaure obtenir un modèle non linéaire affine. Ce modèle a été ensuite utilisé pour la régulation non linéaire par linéarisation entrées/sorties. Les résultats de simulations présentés sont très intéressants et sont validés par les premières expérumentations. Ils nous montrent néanmoins que le découplage des variables d'état est difficile à obtenir lors de la saturation des rapports cycliques moyens Nous avons étudié une méthode afin de lımiter l'effet des saturations et d'obtenir de meilleurs résultats. Enfin, en vue de l'implantation sur un calculateur de type DSP ou PC, nous avons étudié l'effet de la période d'échantillonnage et du temps de calcul de notre algorithme de régulation. Les résultats de la commande par découplage sont corrects jusqu'à une période d'échantillonnage de $125 \mu \mathrm{s}$ en tenant compte du temps de calcul de la commande. Ceci prouve donc la faisabilité de cette commande.

\section{Notations utilisées}

$\begin{array}{lll}p & : \text { nombre de cellules } \\ p_{\mathrm{c}}=p-1 & : \text { nombre de condensateurs flottants } \\ n_{\mathrm{b}} & : \text { nombre de bras } \\ P_{\imath k}^{\star} & : \text { état de la cellule } k \text { du bras } i \\ C_{\imath k} & : \text { condensateur } k \text { du bras } i \\ T_{\imath k}, \bar{T}_{\imath k} & : \text { interrupteurs cellule } k \text { du bras } \imath \\ T_{\mathrm{h}} & : \text { période de découpage ou hachage } \\ V_{\mathrm{s}_{2}} & : \text { tension instantanée aux bornes de la charge du bras } \imath \\ v_{\mathrm{s}_{\imath}} & : \text { tension moyenne aux bornes de la charge du bras } i \\ \imath_{\mathrm{c}_{2 k}} & : \text { courant condensateur } k \text { bras } \imath \\ \imath_{\text {load }} & : \text { courant instantané dans la charge } R, L \\ \imath_{\ell} & : \text { courant moyen dans la charge } R, L \\ V_{\mathrm{c}_{2 k}} & : \text { tension instantanée condensateur } k \text { du bras } i \\ v_{\mathrm{c}_{2 k}} & : \text { tension moyenne condensateur } k \text { bras } i \\ R & : \text { résistance de charge en Ohms } \\ L & : \text { inductance de charge en Henry } \\ X & : \text { vecteur d'état } \\ E_{\mathrm{c}} & : \text { tension d'entrée instantanée (après filtre d'entrée) } \\ e_{\mathrm{c}} & : \text { tension d'entrée moyenne (après filtre d'entrée) }\end{array}$


$E \quad$ : tension d'alimentation continue (avant filtre d'entrée)

$r_{\mathrm{e}} \quad$ : résistance du filtre d'entrée en Ohms

$L_{\mathrm{e}} \quad$ : inductance du filtre d'entrée en Henry

$C_{\mathrm{e}} \quad$ : capacité du condensateur du filtre d'entrée $(\mathrm{F})$.

\section{Bibliographie}

[1] Meynard T.A. and Foch H., Multi-level choppers for high voltage applications, European Power Electronics J. (mars 1992) 45-50.

[2] Foch H. and Meynard T.A., Multi-level conversions: high voltage choppers and voltage source inverters, I.E.E.E Power Electronıcs Specialısts Conference (Toledo, 1992) 397-403.

[3] Meynard T.A. and Foch H., Brevet Francais $\mathrm{N}^{\circ} 91.09582,25$ juillet 91 , Dépôt International PCT (Europe, Japon, U.S.A, Canada) n $92 / 00652$ du 8 juillet 92.

[4] Carrere P., Meynard T.A and Lavieville J.P., 4000 V-300 A eight level IGBT inverter leg, European Power Electronics (Sevilla, 1995) pp. 106-111.

[5] von Westerholt E., Commande non linéaire d'une machine asynchrone, Thèse de doctorat $\mathrm{n}^{\circ} 851$ (INP Toulouse, 1994).

[6] Frick A., von Westerholt E. and dề Fornel B., Nonlinear control of induction motors vıa input-output decoupling, ETPE J. (mars 1993).

[7] Fossard A.J et Normand-Cyrot D., Système non linéaires (Masson, 1993).

[8] Slotine J.J.E. and Li W., Applied non linear Control (Prentice Hall, 1990).

[9] Davancens P. et Meynard T.A., Étude des convertisseurs multi-cellulaires parallèles, première partie : modélisation, $J$. Phys. III France (à paraître).

[10] Gille J.-Ch., Decaulne P. et Pelegrin M., Théorie et calcul des asservissements linéaires (Dunod, 1990).

[11] Le Pioufle B., Gorgiou G. and Louis J.P., Application des commandes non-linéaires pour la régulation en vitesse et en position de la machine synchrone autopilotée, J. Phys. III France 2 (1992) 129-144.

[12] De Luca A. and Ulivi G., Full linearization of induction motors vza nonlinear statefeedback, In Proc. of the 26 IEEE CDC (Los Angeles, 1987).

[13] Oukaour A., Barbot J.P. and Le Pioufle B., Nonlınear control of a variable frequency DC-DC converter, Proc IEEE Conf on Control Applications (Glasgow, 1994).

[14] Schneider H., Conception, étude et réalisation d'un simulateur analogique temps réel en vue de l'application au diagnostic et à l'estimation des variables des systèmes électrotechniques, Thèse de doctorat $\mathbf{n}^{\circ}$ d'ordre : 988 (I.N.P. Toulouse, 1995). 